\title{
A STRATEGY ORIENTED, MACHINE LEARNING APPROACH TO AUTOMATIC QUALITY ASSESSMENT OF WIKIPEDIA ARTICLES
}

\author{
A Thesis \\ presented to \\ the Faculty of California Polytechnic State University, San Luis Obispo
}

In Partial Fulfillment of the Requirements for the Degree Master of Science in Computer Science

by

Gabriel De La Calzada

April 2010 
(C) 2010

Gabriel De La Calzada

ALL RIGHTS RESERVED 


\section{COMMITTEE MEMBERSHIP}

TITLE:

A Strategy Oriented, Machine Learning Approach to Automatic Quality Assessment of Wikipedia Articles

AUTHOR:

Gabriel De La Calzada

DATE SUBMITTED:

April 2010

COMMITTEE CHAIR: Dr. Alex Dekhtyar

Computer Science Associate Professor

Computer Science Department

California Polytechnic State University

COMMITTEE MEMBER: Dr. Franz Kurfess

Computer Science Professor

Computer Science Department

California Polytechnic State University

COMMITTEE MEMBER: Dr. Clark Turner

Computer Science Professor

Computer Science Department

California Polytechnic State University 


\section{ABSTRACT \\ A Strategy Oriented, Machine Learning Approach to Automatic Quality Assessment of Wikipedia Articles \\ Gabriel De La Calzada}

This work discusses an approach to modeling and measuring information quality of Wikipedia articles. The approach is based on the idea that the quality of Wikipedia articles with distinctly different profiles needs to be measured using different information quality models. To implement this approach, a software framework written in the Java language was developed to collect and analyze information of Wikipedia articles.

We report on our initial study, which involved two categories of Wikipedia articles: "stabilized" (those, whose content has not undergone major changes for a significant period of time) and "controversial" (articles that have undergone vandalism, revert wars, or whose content is subject to internal discussions between Wikipedia editors). In addition, we present simple information quality models and compare their performance on a subset of Wikipedia articles with the information quality evaluations provided by human users. Our experiment shows that using special-purpose models for information quality captures user sentiment about Wikipedia articles better than using a single model for both categories of articles. 


\section{ACKNOWLEDGEMENTS}

I would like to thank Franz Kurfess, Clark Turner, Kevin O'Gorman, Mark Hutchenreuther, and Kurt Voelker: Cal Poly professors who kindly allowed us to administer the experimental study in their classes. 


\section{Contents}

List of Tables viii

List of Figures $\quad$ ix

1 Introduction 1

2 Background $\mathbf{5}$

2.1 Wikipedia .................... 5

2.2 MediaWiki API . . . . . . . . . . . . . . . . . . 10

2.2.1 Comparative Reliability Studies . . . . . . . . . 13

2.3 Work On Information Quality . . . . . . . . . . . . . . . . 14

3 Design 20

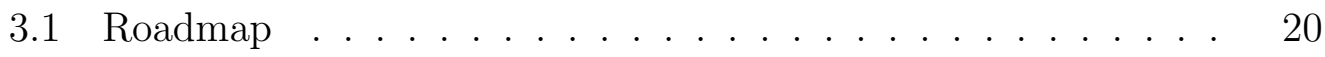

3.2 Wikipedia Models of Quality . . . . . . . . . . . . . 21

3.2.1 Stabilized Article . . . . . . . . . . . . . . . 22

3.2.2 Controversial Article . . . . . . . . . . . . . . . 29

3.2.3 List and Disambiguation Article . . . . . . . . . . . . . 32

3.2.4 Stub Article . . . . . . . . . . . . . . . . 33

3.3 Article Classification .. . . . . . . . . . . . . . . 34

3.4 Goal Specification . . . . . . . . . . . . . . 36

4 Implementation $\quad 38$

4.1 System Architecture . . . . . . . . . . . . . . . . . . 38

4.2 Wikipedia Crawler Foundation Classes . . . . . . . . . . . . 39

4.2.1 classify package . . . . . . . . . . . . 42

4.2.2 qualitymodel package . . . . . . . . . . . . 44

4.2 .3 ir package . . . . . . . . . . . . . . . . . . . 45

4.2.4 wikipediacrawler package . . . . . . . . . . 47

4.2.5 articlefeature package . . . . . . . . . . . 52

5 Validation $\quad 62$

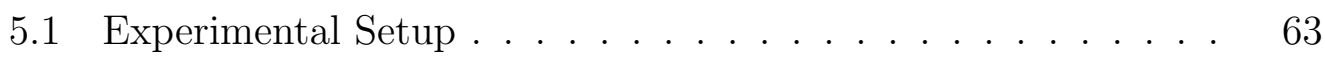

5.1.1 Measures . . . . . . . . . . . . . . . 68

5.1.2 Hypothesis $H 0_{0}$ and $H 0$. . . . . . . . . . . . . . 69

5.1.3 Hypothesis $H 2_{0}, H 2, H 3_{0}$, and $H 3 \ldots \ldots$ 
5.1.4 Hypothesis $H 1_{0}$ and $H 1$. . . . . . . . . . . 70

5.1.5 Miscellaneous ............... . . 70

5.2 Results....................... . . . 70

5.3 Analysis ........................ . . . 75

$\begin{array}{lll}6 & \text { Conclusion and Future Work } & 78\end{array}$

A Student Survey Results $\quad 85$ 


\section{List of Tables}

2.1 Types of MediaWiki API output formats . . . . . . . . . . . . 11

2.2 Query Module Properties . . . . . . . . . . . . . 12

3.1 Stabilized article representation . . . . . . . . . . . . . 24

3.2 Dataset Comparison . . . . . . . . . . . . . . . . 30

3.3 Article representation in the controversial model . . . . . . . . 31

3.4 Article representation of a list/disambiguation article . . . . . 32

3.5 Article representation of a stub . . . . . . . . . . . . 33

3.6 Stabilized Classifier Evaluation . . . . . . . . . . . . . . 35

3.7 Controversial Classifier Evaluation . . . . . . . . . . . . . 35

3.8 List Classifier Evaluation . . . . . . . . . . . . . . . . . . . . 35

3.9 Disambiguation Classifier Evaluation . . . . . . . . . . . . 36

3.10 Stub Classifier Evaluation . . . . . . . . . . . . . . 36

4.1 Top level packages . . . . . . . . . . . . . . . . . . 40

4.2 Top-level classify classes . . . . . . . . . . . . . . . . . . . . . 43

4.3 List of WEKA Machine Learning Algorithms . . . . . . . . . . 44

4.4 Top-level qualitymodel classes . . . . . . . . . . . . . 45

4.5 Top-level ir classes . . . . . . . . . . . . . . . . . . . . . . . 45

4.6 Top-level wikipediacrawler classes . . . . . . . . . . . . . . 47

4.7 Top-level wikipediacrawler.mediawiki classes . . . . . . . . 48

4.8 Top level articlefeture classes . . . . . . . . . . . . . . 53

5.1 Survey Article Classification . . . . . . . . . . . . . . . . . . 64

5.2 Survey Article Quality Scale . . . . . . . . . . . . . . 65

5.3 Survey Confidence Scale . . . . . . . . . . . . . . 65

$5.4 \quad H 0$ : Descriptive statistics . . . . . . . . . . . . . 72

5.5 H0: T-Test Results . . . . . . . . . . . . . . . . 73

5.6 Mean absolute prediction errors . . . . . . . . . . . . 73

5.7 Mixed vs. stabilized, controversial, stub, and list models . . . 73

5.8 T-test results for models within their own articles . . . . . . . 74

5.9 Stabilized \& Controversial Rank Error . . . . . . . . . . . . . 75

A.1 Survey Results $1 \ldots \ldots$. . . . . . . . . . . . 86

A.2 Survey Results $2 \ldots \ldots$. . . . . . . . . . . . . . . . . . . . . . . . . 87

A.3 Survey Results 3 . . . . . . . . . . . . . . . . 88 


\section{List of Figures}

2.1 Article Revision History _. . . . . . . . . . . . . . . . 7

2.2 Watch List. . . . . . . . . . . . . . . . . . . . 7

2.3 Diff Tool . . . . . . . . . . . . . . . . . . . 8

2.4 Recent Changes . . . . . . . . . . . . . . . . . . 8

3.1 Article scoring process . . . . . . . . . . . . . . . . 21

3.2 Euclidean Model vs Mixture Model . . . . . . . . . . . . . 28

4.1 System Architecture . . . . . . . . . . . . . . . . . . . . 39

5.1 Survey Account Creation . . . . . . . . . . . . . . . . . 66

5.2 Main Survey Screen . . . . . . . . . . . . . . . . . . . 67

5.3 Avg Rating vs PageRank . . . . . . . . . . . . . . . . 71

5.4 Avg Rating vs Stabilized Score . . . . . . . . . . . . . . 72

5.5 Avg Rating vs Controversial Score . . . . . . . . . . . . . . . 72 


\section{Chapter 1}

\section{Introduction}

In the past few years, due to significant expansion of its content, and high rankings its articles receive from web search engines, Wikipedia [60] has become the go-to location for a wide range of information for millions of Internet users. Wikipedia is powered by MediaWiki [38], a cross-platform web-based wiki software written in PHP by the Wikimedia Foundation and released under the GPLV2 license. Wikipedia has been the trailblazer for the open content collaborative model of information collection and presentation. One of the goals Wikipedia pursues, outlined by its founder Jimmy Wales is "a world in which every single person on the planet is given free access to the sum of all human knowledge" [61, 39]. Collecting and providing access to a large body

of information, parallels the goals of other encyclopedia. However, Wikipedia takes a distinctly different approach to achieving this task.

The open content collaboration model is a hallmark of Wikipedia. Anyone with an Internet connection can add, remove, or update any content available from Wikipedia. It relies on the collective wisdom of many readers-cum-editors to prevent, and, if necessary, fix, erroneous, false, poorly presented or simply inappropriate content. This is in contrast to the closed development model of traditional written encyclopedias. In the closed development model, only the content which has been rigorously peer reviewed by domain experts is made 
available for public consumption. Therefore, when viewing a Wikipedia article, there's a risk of the article being in an inconsistent or incomplete state. Over the period of Wikipedia's existence, this approach to content creation has been subject to numerous arguments between its critics [54,37] and its defenders $[11,59]$.

Despite potential problems inherent in an open content collaborative model, many people willingly continue to use Wikipedia. Either the users don't realize the potential problems of a open content collaborative model or are placing a great amount of trust in the contributors. When articles can change at any time it may be difficult to determine if an article is of high or low quality.

One way to better understand the quality of an article is to utilize Wikipedia's functional features to follow closely the development of an article, and formulate your own judgment on whether or not an article is good or bad. This is done by examining an article's revision history and figuring out what additions were made or what deletions were made at each revision. This way the user is able to assess how much work the community has put into the article and whether any conflicting contributors are using the target article as a battlefield. Unfortunately this approach is time consuming.

A more practical yet naive approach is for the reader to simply have faith that the contributors of an article are doing a "good job", where good job means the article is complete and any acts of vandalism are cleaned up. However, when we rely on the good will of Wikipedia contributors, we take things for granted and become vulnerable to misinformation.

Methods of quality assessment of large amounts of structured data in data warehouses have been explored in earlier works. However, these techniques don't apply to Wikipedia due to its lack of structure. Fortunately, there is a promising growth of quality assessment research applied to Wikipedia [64, 47, 
24, 52]. However, these works don't make any distinctions between various article types. For example, a highly controversial article is treated the same as one with stabilized activity for purposes of quality assessment.

This work explores the potential of assessing the quality of a Wikipedia article with a two step process based on its type. This proposed approach first discovers an articles' type with a classifier. Finally, a quality model is chosen to evaluate the article based on its type. The intuition behind this approach is that different article types have different definitions of quality. An article type indicates where the article is currently situated along its development phase, the nature of an article's subject, or how Wikipedia structures the article. This work proposes the following major article types: stabilized, evolving, controversial. The focus of this work is on stabilized and controversial articles along with other minor types.

Contributions of this work are as follows:

1. A framework for extraction and analysis of Wikipedia articles. The framework is written in Java, and provides a way to programmatically collect information about articles from Wikipedia or any other web application based on MediaWiki. This framework also provides the groundwork for implementation of article quality models.

2. A series of quality models along with a scoring scheme for Wikipedia articles. The scoring scheme takes a machine learning approach to select an appropriate quality model for any given article.

3. An experiment to measure perceived user quality of Wikipedia articles. In this methodology, perceived user quality of an article is the degree to which users of Wikipedia (experts or not) are able to learn from the article. Our experiment was conducted on a set of 100 Wikipedia 
articles. This experiment consisted of 247 student participants from various Computer Science/Software Engineering courses at California Polytechnic State University, San Luis Obispo. 


\section{Chapter 2}

\section{Background}

\subsection{Wikipedia}

Wikipedia is an example of a globally accessible on-line encyclopedia, where anyone can participate in the preservation of knowledge. This is in contrast to traditional sources of information such as encyclopedias. Wikipedia is an open content project, meaning anyone with an Internet connection can modify or create an article. This openness even allows anonymous, non-registered users to make complete contributions. The motivation behind the open content idea is that as the community works together on content, the content becomes more reliable over time. Consequently, articles found on Wikipedia are never finished as modifications are continuously made. In addition, openness is traded for the lack of formal peer review [18]. Although Wikipedia has come a long way, there is no mechanism for a peer review by subject matter authorities. It's also known that many articles dont cite their primary sources [18].

The open content ideal behind Wikipedia makes vandalism and misinformation possible, and self-interested parties have taken advantage of this in the past. Wikipedia has temporarily banned access from government domains in response to a rising trend of defacement of political candidates [36]. Political operatives have been reported to modify Wikipedia entries to make a certain candidates appear strong or weak. 
To combat misuse of the open content model, Wikipedia provides numerous functional features promoting cooperation between prospective contributors. Contributors have access to an Edit History of every article shown in Figure 2.1. The Edit History allows anyone to view every modification made by everyone to an article since inception [33]. Information on the time, date, and author is provided with each modification listed on the Edit History page. In addition, the Edit History allows users to compare and contrast specific revisions on a line by line basis using the diff tool. An example of the diff tool in action is shown in Figure 2.3. Users who take a particular interest in an article can add that article into a Watch list. When a page is modified in any way, users with that page in their Watch list will be notified on their Watch list page. In the Watch list changes are displayed in descending order relative to modification date. Similar to the Edit History, the Watch list displays information on the time, date, and author. Finally, Wikipedia provides a Recent Changes page displaying any article that has been modified recently [33]. 


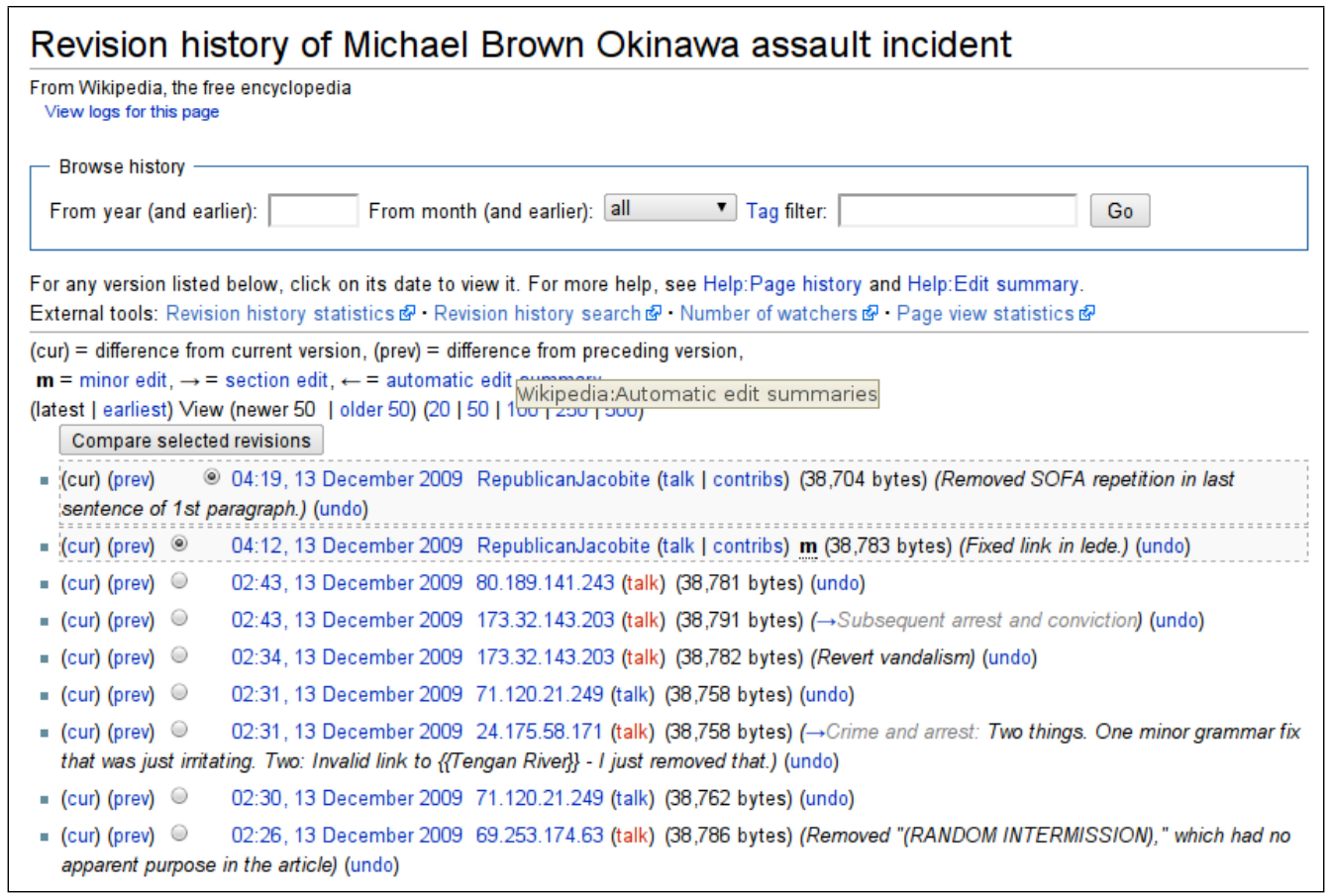

Figure 2.1: Article Revision History

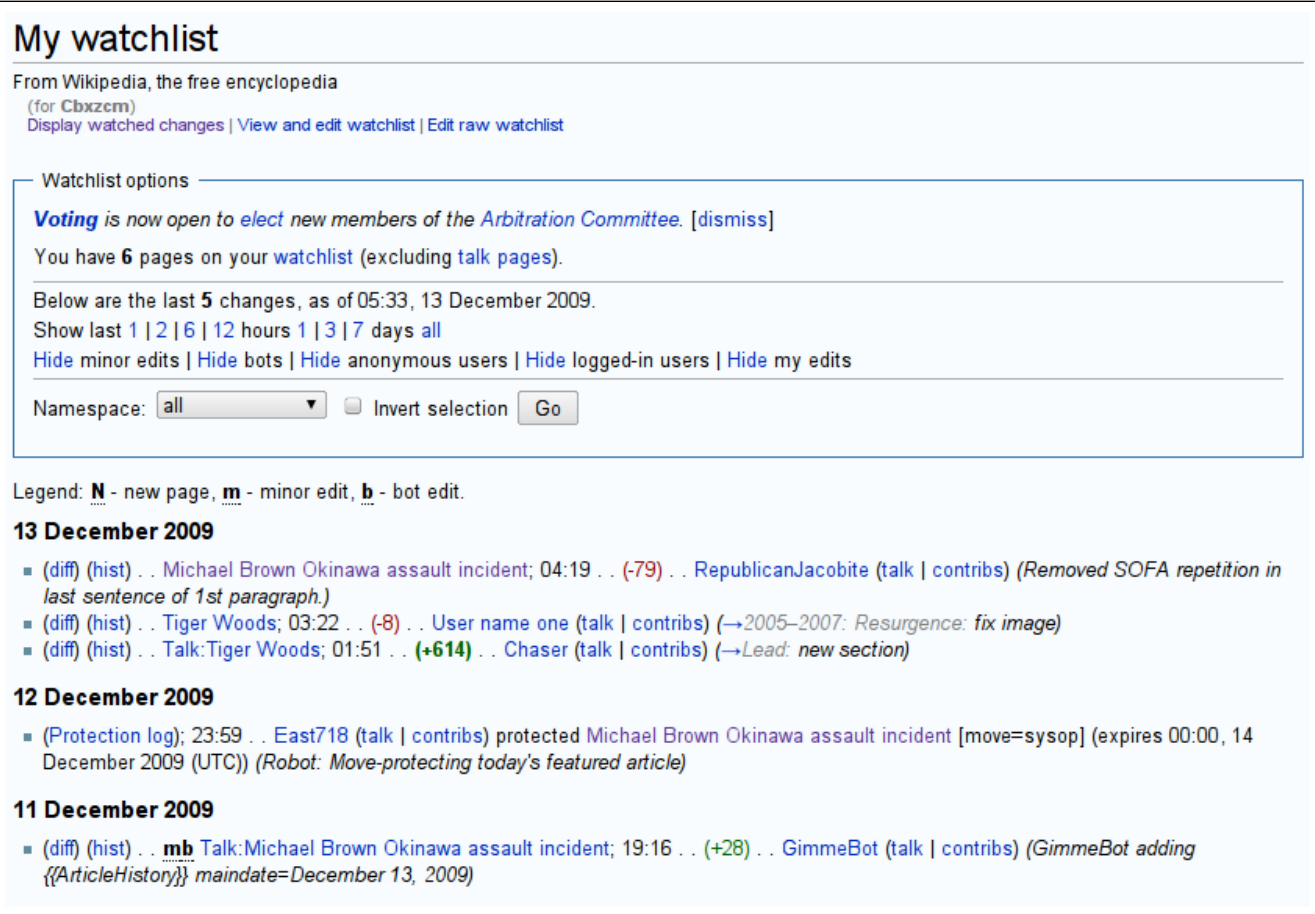

Figure 2.2: Watch List 
tors must follow a Neutral Point of View (NPOV) policy [33]. Contributions to Wikipedia that violate the NPOV policy are reverted or transformed by other contributing users. The NPOV policy was created by Wikipedia founder Jimbo Wales and requires that articles present facts and ideas in a specific manner such that supporters and opponents can agree on equal terms. Wales believes that the functional features of Wikipedia aren't enough to manage open content. The reasoning behind the NPOV policy is that the community which Wikipedia relies on will inevitably reach disagreements on what constitutes knowledge on certain topics. These types of disagreements would result in constant back and forth resubmissions of an article claiming to be correct. Behavior such as this is contradictory to Wikipedias ideal of the community working together to improve the quality of knowledge. Rather than arguing over what is and isn't knowledge, Wikipedia aims to accept all encompassing arguments as human knowledge.

This open content model of information augmented with functional and non-functional defense mechanisms has proven to be successful for Wikipedia. Since its creation in 2001, Wikipedia has grown to a point where its number of articles surpasses all other encyclopedias [53]. During the last three years, Wikipedia has grown to more than 3,000,000 English articles. Each day more than 2,100 articles are submitted by users [22]. Furthermore, its growing popularity has reached international status by expanding to different languages. As of March 2005, Wikipedia contains 195 supported languages [53]. The most populous language on Wikipedia is English, followed by German and Japanese as a close third. Finally, Wikipedia is a free service, intended to support the community in the field of knowledge and research. All Wikipedia content is released under the Creative Commons Attribution/Share-Alike 3.0 licenses. This means that Wikipedia content may be copied, distributed, and displayed 
only if credit is given to the original author(s), and that any derivative content may be distributed under a license similar to the original [1]. Due to the rising popularity and expansion, Wikipedia becomes an ever increasingly attractive source of information for students to cite in their assignments.

\subsection{MediaWiki API}

The work described in this thesis makes much use of the MediaWiki API for data collection. MediaWiki is the wiki software used by Wikipedia. It provides an API that can be used to query Wikipedia for various information. More information about this API is available at http://www.mediawiki.org/ wiki/API. The MediaWiki API for Wikipedia is publicly available at http: //en.wikipedia.org/w/api.php.

The MediaWiki API is accessible through a PHP interface and provides high level access to a MediaWiki application. Access to the API is done through specially crafted URIs for the PHP interface. The parameter list of the URI determine the specifics of the query. The output format of MediaWiki API query is specified in the query and can be one of the following formats shown in Table 2.2. With the MediaWiki API, it's possible to query information from articles, login into the MediaWiki application, and post changes to articles. 


\begin{tabular}{|l|l|}
\hline Name & Description \\
\hline JSON & JavaScript Object Notation \\
\hline PHP & Hypertext Pre-processor \\
\hline WDDX & Web Distributed Data eXchange \\
\hline XML & Extensible Markup Language \\
\hline YAML & YAML Ain't Markup Language \\
\hline
\end{tabular}

Table 2.1: Types of MediaWiki API output formats

Organization of the MediaWiki API is broken into modules. What follows is a summary of a number of noteworthy modules.

Site Matrix. The Site Matrix module is used for obtaining a list of all MediaWiki instances accessible through MediaWiki API. For example, the English, Dutch, and French editions of Wikipedia are accessible through Wikipedia's MediaWiki API site matrix module.

Open Search. The open search module provides the functionality for searching articles within a MediaWiki application. A search is specified with a search string, and may be controlled by limiting the search results, and restricting the MediaWiki namespaces to search.

Click Tracking. The Click tracking module allows for tracking of user mouse clicks on Javascript elements.

Login/Logout. The login and logout module provides functionality for logging in and out with a username and password into a MediaWiki application as shown in the site matrix. 
Query Module. The "Query" module is the most involved module of the MediaWiki API and provides the mechanisms for retrieving information about the MediaWiki application and its articles. With the query module, groups of articles can be queried given their title, page id, or revision id. The title is a case sensitive string representation of an article title. Page ID is a unique number which is assigned to a article title. Finally, revision ID is a unique number assigned to a particular combination of article title and revision.

The types of article information that can be retrieved are divided into article properties shown in Table 2.2. Most of these properties are self-explanatory. However, the "links" property refers to hyperlinks in an article pointing to another article within the same MediaWiki application. On the other hand, "external links" refers to hyperlinks in an article which point to a location outside of the MediaWiki application. For fine grained information retrieval, each property is broken into sub-properties. For instance, the revision property is broken into the following sub-properties: revision id, flags timestamp, author, size (in bytes), user comment, and revision content.

\begin{tabular}{|l|l|}
\hline Name & Description \\
\hline Revision & A single submission by a single author \\
\hline Internal Link & $\begin{array}{l}\text { A link from one article to another arti- } \\
\text { cle }\end{array}$ \\
\hline Template & $\begin{array}{l}\text { A premade box-like structure contain- } \\
\text { ing information }\end{array}$ \\
\hline Language Link & $\begin{array}{l}\text { An internal link to the same article but } \\
\text { in a different language }\end{array}$ \\
\hline Image & A binary image included in the article \\
\hline Category & An organizational label of an article \\
\hline External Link & $\begin{array}{l}\text { A link pointing to a location outside of } \\
\text { the current MediaWiki application. }\end{array}$ \\
\hline
\end{tabular}

Table 2.2: Query Module Properties

Access to each of these modules can be controlled by maintainers of the 
MediaWiki application. Each account type can be assigned different access privileges. For example, in Wikipedia anonymous users are allowed to receive up to 500 revisions of an article per request, while accounts flagged with "bot" privileges can can receive up to 5000 revisions per request.

\subsubsection{Comparative Reliability Studies}

Ever since Wikipedia's introduction, numerous studies comparing Wikipedia to traditional sources of knowledge have been conducted, as documented in [62]. The majority of these studies compare Wikipedia to an authority such as traditional peer-reviewed sources or a team of experts.

The results from a number of studies suggest that Wikipedia suffers from major errors of omission. The study conducted by [12] analyzed Wikipedia articles for seven top Western philosophers. These articles where then compared to a consensus list of themes acquired from various works in philosophy. From this comparison, it was found that the Wikipedia articles on average covered only $52 \%$ of the list of themes. However, no errors were found in the content of these articles.

Similarly, in [15] a research team analyzed 80 Wikipedia articles on drugs. They found that the articles often missed important information and a small number of factual errors.

On the other hand, a number of studies suggest that Wikipedia is no worse or if not, even better than existing peer reviewed sources of information. In [6], 50 Wikipedia articles were compared to their counterparts in a German

encyclopedia "Brockhaus Enzyklopädie" [4]. Results showed that on average Wikipedia articles were more accurate, complete and up to date, while the Brockhaus articles were judged to be more clearly written. 
A number of other studies [5, 23, 43, 7] compared the content of selected Wikipedia articles to other encyclopedia, including Encarta and Encyclopedia Britannica. These comparisons did judge Wikipedia to be less reliable than the traditional encyclopedia.

Comparative studies help "calibrate" the public perception of the quality and reliability of Wikipedia in general. However, these studies involve tiny (and not always representative) portions of the Wikipedia. Additionally, while Wikipedia itself relies on achieving quality through article evolution, comparative studies mimic the validation procedures used by conventional encyclopedia. Our work described in the paper uses human assessment of article quality, but relies on peer assessment rather than expert reviews.

\subsection{Work On Information Quality}

The area of information systems contains a large body of research on the definition of quality of information systems based on its problem context.

A framework for defining data quality of a specific domain from a consumer point of view was proposed in [57]. This framework views data quality in the following dimensions: intrinsic, contextual, representational, and accessibility. $[55,35]$ proposes that data quality is multi-dimensional, then identifies and proposes rigorous definitions for these dimensions. Dimensions are broken into two hierarchies: internal and external. Internal quality deals with the design and operation of the data warehouse, while external quality deals with the use and value. Internal and external quality is further sub-divided into data and system related dimensions. Data related dimensions involve the actual content

of the data, while system related dimensions involve the system hosting the warehouse. 
Frameworks and methodologies for quality assessment of information systems have also been proposed. For multi-user information-decision systems, [10], proposes a quality model based on data-flow processing, and an automatic mechanism for detecting quality errors. [27] introduces a domain independent framework for establishing data quality of a data warehouse. This approach involves correlating semantic models of data warehouse architecture to a higher level model of quality. [68] also organizes quality dimensions into intrinsic, contextual, representational, and accessibility quality. However, [68] proposes a different set of dimensions for each category. [32] introduces a framework for manually assessing the quality of information of data warehouses. In this framework, quality dimensions are organized in terms of consumer expectations and conformance to specifications. Measurement of information quality is conducted through a consumer survey (IQA instrument) and gap analysis methods.

The techniques for measuring quality of data warehouses aren't a direct fit in the context of Wikipedia for several reasons. This is because these techniques leverage the structure of data of a data warehouse. However, "data" in Wikipedia is highly unstructured. In Wikipedia each article is collection of a unstructured text. Therefore, the quality assessment of Wikipedia articles requires a different approaches.

This area of research for Wikipedia quality is fairly new and therefore there isn't a sizable body of research. In [25], the authors proposed and evaluated four different quality models: Naive, Basic, PeerReview, and ProbReview. In the Naive model, the quality of an article is directly proportional to the number of words contained in that article. The Basic model co-opts the HITS framework [29], which determines the hub and authority scores of web pages, to the problem of estimation of the quality of Wikipedia articles. The higher 
the authority of the authors of an article, the higher is the quality of that article. Authority of a user is based on the quality of the articles that user has authored. Both article quality and user authority enforce each other. Equations 2.1 and 2.2 model quality in the Basic model, where $A_{j}$ is the authority of user $u_{j}$.

$$
\begin{aligned}
& Q_{i}=\sum_{j} c_{i j} A_{j} \\
& A_{j}=\sum_{i} c_{i j} Q_{i}
\end{aligned}
$$

The third model, PeerReview, identifies a separate quality of each word in an article. Quality of a word is based on the authority of the user who authored the word, and the authority of any user who reviewed the word. This approach, thus, rewards words that survived multiple review cycles. The authority of a user is based on the quality of the words the user has authored or reviewed. The sum of word qualities belonging to a single article is interpreted as the overall quality of the article. Equations 2.3 and 2.4 model quality in the PeerReview model, where $q_{i k}$ is the quality of the $k^{\text {th }}$ word in article $a_{i}$, $w_{i k}$ the $k^{\text {th }}$ word in article $a_{i}, \stackrel{\mathrm{A}}{\leftarrow} u_{j}$ the set of words authored by user $u_{j}$, and $w_{i k} \stackrel{\mathrm{R}}{\leftarrow} u_{j}$ the set set words reviewed by user $u_{j}$.

$$
\begin{aligned}
q_{i k} & =\sum_{w_{i k} \stackrel{\mathrm{A}}{\leftarrow} u_{j} \cup w_{i k} \stackrel{\mathrm{R}}{\leftarrow} u_{j}} A_{j} \\
A_{j} & =\sum_{w_{i k} \stackrel{\mathrm{A}}{\leftarrow} u_{j} \cup w_{i k} \stackrel{\mathrm{R}}{\leftarrow} u_{j}} q_{i k}
\end{aligned}
$$

The ProbReview model assumes that a user who submits a revision to an article does not necessarily review every word in that submission. For example, a user skimming through an article might notice that certain statistics 
are missing from the article and submits a revision which contains the original article content in addition to the new statistics. In this case, the new statistics were authored, however the remaining content wasn't reviewed. The ProbReview model is a modification of the PeerReview model. It takes into account the probability that a user submitting a revision has reviewed a word in a document. Equations 2.5, 2.6, and 2.7 model quality in the ProbReview model. Function Prob determines the probability that user $u_{j}$ reviewed the word $w_{i k}$. The intuition behind this function is that when a user authors content of an article, that user is more likely to review content located closer to the newly authored content.

$$
\begin{gathered}
q_{i k}=\sum_{j} f\left(w_{i k}, u_{j}\right) A_{j} \\
A_{j}=\sum_{i, k} f\left(w_{i k}, u_{j}\right) q_{i k} \\
f\left(w_{i k}, u_{j}\right)= \begin{cases}1 & \text { if } w_{i k} \stackrel{\mathrm{A}}{\leftarrow} u_{j} \\
\operatorname{Prob}\left(w_{i k} \stackrel{\mathrm{R}}{\leftarrow} u_{j}\right) & \text { otherwise }\end{cases}
\end{gathered}
$$

A study by Lih[33] focuses on the "reputation" of an article. Lih's model assumes that the more reputable an article, the higher its quality. Reputation in this context is the amount of collaborative work that went into the authoring of an article. Instead of focusing on the actual content of an article for quality assessment, Lih's methodology focuses only on an article's meta data. Specifically, the model relies on information found directly in an article's revision history. In this model, rigor is defined as the total number of revisions to a particular article. The assumption is that the more revisions an of an article, the deeper the treatment of the subject and higher scrutiny on the content. Diversity is defined as the total number of unique users contributing 
to an article. The assumption is that more unique contributors means more voices and different points of view on the subject of a given article. Articles whose rigor and diversity are both above the media are considered to be of high quality.

Zeng et al.[64] propose a quality model which focuses on the trustworthiness of an article. This model recognizes that articles evolve over time, and thus their trustworthiness evolves over time. An article that was trustworthy a month ago might not be trustworthy today. The trust of an article is based on the trust of the previous version of the article, the trust of the current author, and any insertions or deletions. Trust is a continuous number ranging from $[0,1]$, where a trust of 0 is most untrustworthy while a trust of 1 is most trustworthy. This model uses a dynamic Bayesian network to model trust.

In [47], the authors used machine learning to construct an automated quality assessment system. The authors identified six quality classes of articles from worst to best: stub, B-article, good article, A-article, and featured article. A classifier based on the maximum entropy model was then used to predict which of these quality classes an article belongs to. The classifier made use of over 50 features which fell into one of the following four categories: length measures, part-of-speech usage, web-specific features, and readability metrics.

In [24], Dalip utilized the same machine learning approach as [47] to assess Wikipedia article quality. However, Dalip treats the problem of automatic quality assessment of Wikipedia articles as a regression analysis problem and uses a support vector regression classifier to solve it [52]. The classifier uses the quality classes from [47]. Thus, an article predicted as "stub" is assigned stub quality while an article predicated as "Featured-Article" is assigned featured article quality.

Our approach to evaluating the information quality of a Wikipedia arti- 
cle is similar to the approaches described in this section. We use a variety of information about an article to develop models for predicting its quality. However, whereas all work described above uses one quality assessment/prediction model for all Wikipedia pages, we investigate a two-tier approach in which we first determine a broad category of a given article, and then use categoryspecific quality prediction model to compute the information quality estimate. Additionally, we validate our models and our approach empirically, by investigating, how well they predict the quality assessments made by casual Wikipedia visitors. 


\section{Chapter 3}

\section{Design}

\section{$3.1 \quad$ Roadmap}

The design and implementation of the automatic quality assessment system is guided by the following roadmap:

1. Identification of various Wikipedia article types and the design of quality models for each type. In this work, we focus on the following article types: stabilized, controversial, stub, list, and disambiguation.

2. Design and implementation of a software framework for automatic quality assessment of Wikipedia articles.

3. Design of a Wikipedia article scoring mechanism which unifies all quality models for each identified Wikipedia article classification.

4. Design of a Wikipedia automatic quality assessment system which makes use of the quality scoring mechanism and software framework.

First, a quality model is designed for each classification of a Wikipedia article. Each model is designed to leverage the characteristics of their respective article classification, and is used as part of an article's final assessment. Next is the design and implementation of a automatic quality assessment framework. 
This framework handles the retrieval and analysis of Wikipedia article information. Finally, the framework is used to build a quality assessment system on these models.

\subsection{Wikipedia Models of Quality}

We propose a two-step approach to evaluating and/or prediction the information quality of Wikipedia articles. First, we separate Wikipedia articles into a number of categories, based on their history and the nature of their topics. Unlike [24] and [47], which split Wikipedia articles horizontally by the perceived quality, we split the articles vertically: articles belong to the same category if they exhibit similar properties, not if they are of similar quality. On the second step, we develop a quality prediction model for articles within each category and apply it to estimating the information quality of the articles.

A visualization of our implementation to this approach is shown in Figure 3.1. In the first step an article is given as input to a series of trained classifiers. These classifiers predict whether or not an article belongs to a defined article category. In the second step, the appropriate quality models are applied and combined for a final quality assessment metric. A more detailed explanation of this process is described in Section 3.3.

Classifier

Figure 3.1: Article scoring process 
We have identified various types of Wikipedia articles. The first set of article types pertain to the degree of inclusion of the subjects body of knowledge. The First of these types is "stabilized". Stabilized types are articles which exhibit few minor revisions due to the inclusion of most of the content. In contrast, evolving types exhibit numerous major revisions which contribute to the content.

Next are types which are specific to an article's subject. Controversial types involve subjects which are highly interpretive. This high degree of interpretation results in splits between the community. Types which involve subjects which inherently require constant revisions are identified as volatile. An article on the current president of the United States may be considered volatile, while an article on Benjamin Franklin would be considered non-volatile.

Finally, there are types which are based on the organizational structure of Wikipedia. These types include stubs, list, and disambiguation types. Stubs are short placeholders marked as incomplete. List types act as a directory of to other links related by subject. Disambiguation pages help a reader locate a specific article among other phonetically similar articles.

In this thesis we define quality models for two of the most difficult types: controversial and stabilized. In addition, we define quality models for stub, list, and disambiguation types because they are straightforward. Quality models for the remaining identified types is a candidate for future work.

\subsubsection{Stabilized Article}

Informally, a stabilized Wikipedia article is one that has more or less "caught up" with the total knowledge of the topic and is considered to be complete content-wise. Stabilized article topics, typically, refer to events, people, no- 
tions, etc., that no longer change over time. The changes to these types of articles are mostly either "maintenance" revisions, such as those made by automated bots to update the categories of articles, or the reverts of a random vandalism attack. Since a stabilized article is supposed to be complete contentwise, we expect in general to find significant accuracy of the content relative to the total topic knowledge.

For this category of articles, we currently have implemented the following types of quality models: a model based on Blumenstock's observations [26], a model based on Euclidean distance from the "Featured" cluster, and a model based on a mixture model of a sample of featured articles. The quality models presented in this paper for stabilized articles can be classified into two categories: featured article based and non-featured article based. The featured article based models utilize Wikipedia's notion of a "Featured Article" as a standard of quality. On the other hand, non-featured article models don't use this assumption. [46]

Wikipedia's criteria for a "featured" article are very compatible with our definition of a stabilized article. Specifically, Wikipedia's policy mandates that featured articles must be stable: "It is not subject to ongoing edit wars and its content does not change significantly from day to day, except in response to the featured article process" [63]. These types of models thus measure how "similar" some arbitrary article is to existing featured articles. These models require a sample of existing featured articles as a point of reference. The following two models differ on how this sample of featured articles is interpreted as a point of reference. 


\section{Euclidean Distance Model}

This model is essentially an application of the concept of clusters within information retrieval. In the Euclidean distance model, articles are represented by the features shown in Table 3.2.1. For a more detailed descriptions of each article feature please refer to Section 4.2.5.

\begin{tabular}{|l|l|}
\hline Feature Name & Description \\
\hline Log Length & Base 10 logarithm of article length in bytes \\
\hline Citation Density & Citations per article length \\
\hline Internal Link Density & Internal links per article length \\
\hline External Link Density & External links per article length \\
\hline Image Count Density & Images per article length \\
\hline Section Count Density & Sections per article length \\
\hline
\end{tabular}

Table 3.1: Stabilized article representation

Furthermore, this model has a "training" component. Before this model can be used, a sample of featured articles must be collected. Each article within this sample is then put into a "featured article cluster". In information retrieval, a cluster contains entities which aren't exactly equal but related. In our case, any two featured articles may have different vector values but are related in that they are both deemed a "featured article" by the Wikipedia community.

Once a featured article cluster is created, the center of the cluster is determined. In data mining literature, there are numerous ways to determine a cluster's center. For example, in the kmeans clustering algorithm [50], a cluster's center is defined to be the average of all entities within the cluster. However, for this model the median of all entities is used as the center. The median rather than the average was used because of the sheer amount of diver- 
sity of featured articles found on Wikipedia. Computing the median of each article's vector is less likely to be affected by such outlier behavior.

When the center of the featured article cluster is determined, the quality measure of some arbitrary article is straightforward. First, the target article is put into a vector representation consisting of the above features. Next, the Euclidean distance between the target article's vector and the featured article center is determined. The equation for Euclidean distance between two points $p$ and $q$ is shown in Equation 3.1. A smaller distance between the target article and featured cluster is interpreted as the highest quality stabilized article. On the other hand, the larger the distance between the two, the lower the quality of the target article.

$$
d(p, q)=\sqrt{\sum_{i=1}^{n}\left(p_{i}-q_{i}\right)^{2}}
$$

\section{Mixture-Model Model}

This model is based on the intuition that all of the above article measures (except for length) can be considered as necessary building blocks for an article. For instance, images, references, citations, paragraphs, and links are all hallmarks of a quality article. However, too much or too little of these building blocks can cause an article to be over-developed or under-developed. Quality in this model is based on the article length. A higher length indicates a higher article quality. However, the expressive power of article length is further scaled up or down based on the remaining article measures. When an article has the exact same proportion of characteristics to the "typical" featured article then the expressive power of article length is at its highest. However, as an article's 
characteristics deviates from the that of a typical featured article, then the expressive power of article length diminishes. In this model, each Wikipedia article is represented by the features shown in Table 3.2.1. For a more detailed explanation of the features, please refer to Section 4.2.5.

Similar to the Euclidean distance model, this model requires a sample of featured articles from Wikipedia. However, instead of interpreting the sample of featured articles as a cluster, the sample is interpreted as a collection of mixture components of a mixture model. Within this mixture model are six mixture components derived from the sample set of featured articles. These mixture components are: Gaussian probability density functions for logarithm of length, citation density, internal link density, external link density, image count density, and section count density.

The equation for some arbitrary mixture component $i$ is shown in Equation 3.2 , where $\mu$ is the mean value for the component and $\sigma$ is the standard deviation. For example, a "length in bytes" component represents a Gaussian probability density function for the length in bytes of a sample of featured articles. Within each mixture component, the standard deviation $\sigma$ is multiplied by a "forgiveness factor". This forgiveness factor controls how strict or lenient the component is. A default factor of 2 is used in this model.

$$
C_{i}(\text { article })={\frac{1}{\sqrt{2 \pi \sigma^{2}}}}^{-\frac{(x-\mu)^{2}}{2 \sigma^{2}}}
$$

To evaluate the quality of an article, the sum of each component is performed. The sum of each component is then multiplied by a normalization factor. This normalization factor is equal to the sum of the maximum of each mixture component. With this normalization factor, a resulting value ranging from 0 to 1 inclusively is guaranteed. The final formula is shown in Equation 3.3 . 


$$
q(\text { article })=\operatorname{length}(\text { article }) \times \frac{\sum C_{i}(\text { article })}{\sum C \max _{i}}
$$

\section{Stabilized Model Comparison}

By Wikipedia's featured article policy, a featured article should be the pinnacle of article quality. On the other hand, an average article should have lower quality. Furthermore, both of the above models are compared with each other. Recall that the output of the mixture-model model is proportional to article quality and the output of the Euclidean model is inversely proportional to article quality. Therefore, in order for both models to be consistent with each other the Euclidean model should output big values while the mixture-model model outputs small values for "bad" articles. Likewise, the Euclidean model outputs small values and the mixture-model model outputs large values for "good" articles.

For comparison, a number of random featured articles and random articles were chosen. Both types of articles were chosen through the use of the MediaWiki API. Within Wikipedia is an article listing all previous featured articles. The MediaWiki API was used to retrieve the internal links of this page. These links were then randomized using a pseudo random number generator, followed by the selection of articles. The selection of all random articles followed a similar procedure. The MediaWiki API was used to generate a list of random articles from the "Main" namespace.

Next, both the Euclidean model and mixture-model model were used to evaluate all articles within the random featured article set and random article set. The purpose of this step is to analyze the relationship between both models. Results of this step are shown in Figure 3.2. 
Figure 3.2 shows the results of plotting the quality measures of the Euclidean model vs the measures of the Mixture model. The x-axis represents the Euclidean model while the y-axis represents the Mixture model. There are 2200 points in this plot, and each point represents a single article in the dataset. Green dots indicate featured articles, while red points indicate a randomly selected article. Note that a lower value for the Euclidean model is an indication of higher quality. On the other hand, a higher value for the Mixture model indicates high quality.

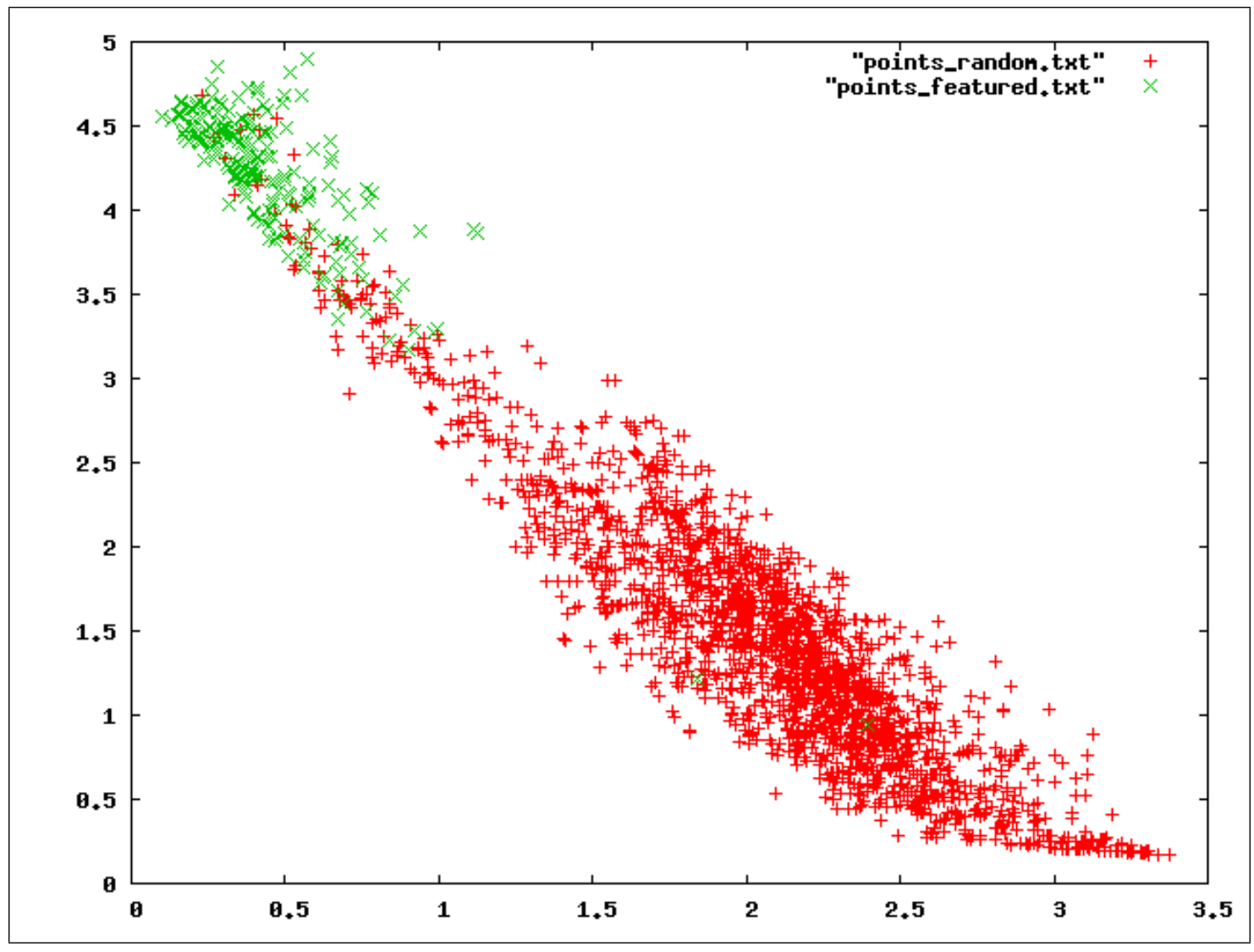

Figure 3.2: Euclidean Model vs Mixture Model

As shown in Figure 3.2.1, there is a noticeable difference between featured articles and an average article. One difference that stands out the most is article length in bytes. The mean length of featured articles is approximately 
one order of magnitude large than the mean length of random articles. This makes sense because featured articles tend to be more complete and information rich. Another interesting difference is in the number of sections. The number of sections in the featured article set is about an order of magnitude more than the number of sections in the random article set. This discrepancy indicates that featured articles are more structured and organized than random articles. Another interesting difference is in the number of sections. The number of sections in the featured article set is about an order of magnitude more than the number of sections in the random article set. This discrepancy indicates that featured articles are more structured and organized than random articles. Finally, the set of featured articles had a significant amount of citations compared to the set of random articles.

As shown in Figure 3.2, the further an article is from the "Featured article" cluster, the lower its score according to the mixture-model model. Likewise, the closer an article is to the featured cluster, the higher its score according to the mixture-model model.

\subsubsection{Controversial Article}

Controversial articles are articles whose topic or content are subject to a range of opinions. Wikipedia editorial policy requires neutral point of view narratives, but Wikipedia editors are human, and, on occasion, their biases make it into the text of the articles they edit, intentionally or unintentionally. When other editors detect such biases and disagree with them, the article may become a subject to controversy. Some articles are inherently controversial due to the nature of their topic and content (for example the article on Religion). Other articles may be going through a controversial phase due to certain attention- 


\begin{tabular}{|l|l|l|}
\hline & 200 Random Featured Articles & 2000 Random Articles \\
\hline Length & & \\
\hline Average & 49068.29 & 4678.66 \\
\hline Median & 44491 & 2283.5 \\
\hline Min & 2106 & 0 \\
\hline Max & 148333 & 262000 \\
\hline Section Count & & \\
\hline Average & 17.47 & \\
\hline Median & 16 & 4.64 \\
\hline Min & 5 & 3 \\
\hline Max & 48 & 1 \\
\hline & & 52 \\
\hline Citation Count & & \\
\hline Average & 96.86 & 3.71 \\
\hline Median & 87 & 0 \\
\hline Min & 0 & 0 \\
\hline Max & 558 & 13 \\
\hline Images & & \\
\hline Average & 13.73 & 2.38 \\
\hline Median & 11 & 2 \\
\hline Min & 1 & 0 \\
\hline Max & 69 & 56 \\
\hline & & \\
\hline Word Count & & 601.21 \\
\hline Average & 6362.07 & 272 \\
\hline Median & 5890.5 & 0 \\
\hline Min & 194 & 20936 \\
\hline Max & 21654 & \\
\hline & & \\
\hline
\end{tabular}

Table 3.2: Dataset Comparison 
grabbing current event or other circumstances. Controversial articles are often the target of vandalism and act as a battleground for revert wars. Historically, a controversial article can be characterized by large number of reverts due to vandalism and revert wars and a high number of anonymous contributions.

Controversial articles are very frequently edited by users and are often targets of revert wars or vandalism. Quality assessment of a controversial article thus takes into account the article revision history. The quality model for controversial articles is based on the mixture model family used by stabilized articles. In contrast to stabilized articles, the controversial article mixture model makes uses of historical mixture components. Each Wikipedia article is represented by the Features shown in Table 3.2.2. For a more detailed description of article features, please refer to Section 4.2.5.

\begin{tabular}{|l|l|}
\hline Feature Name & Description \\
\hline Avg. Number of Reverts & $\begin{array}{l}\text { Average number of reverts in the arti- } \\
\text { cle's revision history }\end{array}$ \\
\hline Revisions Per Registered User & $\begin{array}{l}\text { Average revisions per registered au- } \\
\text { thors }\end{array}$ \\
\hline Revisions Per Anonymous User & $\begin{array}{l}\text { Average revisions per anonymous au- } \\
\text { thors }\end{array}$ \\
\hline Percentage of Anonymous Users & Percentage of anonymous authors \\
\hline
\end{tabular}

Table 3.3: Article representation in the controversial model

The quality model for controversial articles is nearly identical to the mixturemodel model for stabilized articles. However, the controversial model uses mixture components shown in Table 3.2.2. Each component is a Gaussian probability distribution shown in Equation 3.4. The final quality score takes into account all mixtures components and is shown in Equation 3.5.

$$
C_{i}(\text { article })={\frac{1}{\sqrt{2 \pi \sigma^{2}}}}^{-\frac{(x-\mu)^{2}}{2 \sigma^{2}}}
$$




$$
q(\text { article })=\frac{\sum C_{i}(\text { article })}{\sum \operatorname{Cmax}_{i}}
$$

\subsubsection{List and Disambiguation Article}

List articles tend to act as a directory of Wikipedia articles related by some specified subject. For example, the Wikipedia article "List of mountains by elevation" contains Wikipedia links to known mountains sorted by maximum elevation. Consequently, a list article is structurally similar to a disambiguation article. Within Wikipedia, list articles typically belong to a list category or possess a title beginning with some variant of "List of".

Disambiguation articles have only one purpose: to guide readers from an ambiguous search article to their specific article of interest. For instance, when a user search for "Python" at Wikipedia, the user is shown the Python disambiguation article. The Python disambiguation article helps readers distinguish between Python the programming language, a species of animal, or the Monty Python comedy troupe. Structurally, disambiguation articles are typically categorized by a high ratio of links to other Wikipedia pages to textual content. Furthermore, the titles of links in a disambiguation page tend to share one or two words in common. Within Wikipedia, a disambiguation articles typically has a "(disambiguation)" string in its title or is categorized under a disambiguation category. A list/disambiguation article is represented by the features shown in 3.4 .

\begin{tabular}{|l|l|}
\hline Feature Name & Description \\
\hline Revealing Title & $\begin{array}{l}\text { Whether or not the article has a "List } \\
\text { of" or " (disambiguation)" in its title }\end{array}$ \\
\hline Revealing Category & $\begin{array}{l}\text { Whether or not an article belongs to a } \\
\text { "List of" or "disambiguation" category }\end{array}$ \\
\hline Internal links to total content & Ratio of internal links to total content \\
\hline
\end{tabular}

Table 3.4: Article representation of a list/disambiguation article 
The purpose of list and disambiguation articles is to provide a collection of internal links to other MediaWiki articles. Based on this definition, article quality takes into account its link structure. Such an article is high quality if the links it provides are highly ranked. The quality of a list and disambiguation article is defined as its PageRank value times its number of links as shown in Equation 3.6.

$$
q(\text { article })=\frac{\text { PageRank }(\text { article })}{100} \times \text { NormInternalLinks }(\text { article })
$$

\subsubsection{Stub Article}

A stub article exists only as an empty/incomplete placeholder. These types of articles are obviously a major work in progress and require effort from the Wikipedia community. Structurally, stub articles can be characterized by low article size in bytes. Historically, stub articles have very few revisions from a small amount of authors. Usually, such an article is marked by the community as being a stub. For instance, a stub article might have "(stub)" in its title or belong to a special stub category. Stub types of articles are obviously a major work in progress and requires effort from the Wikipedia community.

\begin{tabular}{|l|l|}
\hline Feature Name & Description \\
\hline Length & Size in bytes of the current revision \\
\hline Revision Count & $\begin{array}{l}\text { Total number of entries in the revision } \\
\text { history }\end{array}$ \\
\hline Stub Category & $\begin{array}{l}\text { Whether or not the article is filed under } \\
\text { a stub category }\end{array}$ \\
\hline Unique Editor Count & $\begin{array}{l}\text { Total number of unique editors in the } \\
\text { revision history }\end{array}$ \\
\hline
\end{tabular}

Table 3.5: Article representation of a stub 
Stub articles are by definition incomplete. The quality of stub articles is always the lowest $(0)$.

$$
q(\text { article })=\log _{2}(\text { length }(\text { article }))
$$

\subsection{Article Classification}

Before the quality model for either stabilized or controversial articles can be applied to a given Wikipedia article, we must first determine if an article is stabilized or controversial (or if it belongs to a different category). We achieve this using supervised learning (classification) techniques. In particular, for each article category, we develop and train a classifier. Given a Wikipedia article, finding its quality is a two-step process as shown in Figure 3.1. First, the article's features are extracted, and are run against a battery of classifiers (only two for the experiments described in this paper). When a classifier in the series positively classifies the target article, a quality model corresponding to the classifier type is applied to the article. For the case where a target article is classified as positive by multiple classifiers in the series, the average of outputs for each applied quality model is used as the final score of the target article. Finally, for the case where the target article is not positively classified by any classifier in the series, the stabilized model of article quality is utilized as the final score. In the experiments described in this thesis, we only consider articles that were positively classified by at least one of our classifiers.

Each classifier was trained from a dataset of 96 Wikipedia articles. This dataset was manually chosen to include a mix of each article type described in the previous sections. Class labels for this dataset were manually assigned. A number of supervised learning algorithms provided by WEKA [40] were then 
utilized to build classifiers for this dataset. Among these algorithms, the one which provided the best results was chosen as the algorithm for the final classifier. In this case, the sequential minimal optimization (SMO) $[42,28]$ learning algorithm for training a support vector machine classifier was chosen. Using leave-one-out cross validation, the precision (percentage of correct predictions) and recall (percentage of coverage) for the SMO classifiers for stabilized and controversial articles are shown in Tables 3.3 and 3.3.

\begin{tabular}{|l|l|l|l|}
\hline & Precision & Recall & Class \\
\hline & 0.800 & 0.784 & false \\
\hline & 0.761 & 0.778 & true \\
\hline Weighted Avg. & 0.782 & 0.781 & \\
\hline
\end{tabular}

Table 3.6: Stabilized Classifier Evaluation

\begin{tabular}{|l|l|l|l|}
\hline & Precision & Recall & Class \\
\hline & 0.972 & 0.920 & false \\
\hline & 0.760 & 0.905 & true \\
\hline Weighted Avg. & 0.925 & 0.917 & \\
\hline
\end{tabular}

Table 3.7: Controversial Classifier Evaluation

\begin{tabular}{|l|l|l|l|}
\hline & Precision & Recall & Class \\
\hline & 0.989 & 1.00 & false \\
\hline & 1.00 & 0.800 & true \\
\hline Weighted Avg. & 0.990 & 0.990 & \\
\hline
\end{tabular}

Table 3.8: List Classifier Evaluation 


\begin{tabular}{|l|l|l|l|}
\hline & Precision & Recall & Class \\
\hline & 1.00 & 1.00 & false \\
\hline & 1.00 & 1.00 & true \\
\hline Weighted Avg. & 1.00 & 1.00 & \\
\hline
\end{tabular}

Table 3.9: Disambiguation Classifier Evaluation

\begin{tabular}{|l|l|l|l|}
\hline & Precision & Recall & Class \\
\hline & 0.921 & 0.986 & false \\
\hline & 0.950 & 0.760 & true \\
\hline Weighted Avg. & 0.929 & 0.927 & \\
\hline
\end{tabular}

Table 3.10: Stub Classifier Evaluation

\subsection{Goal Specification}

To support automatic quality assessment of Wikipedia articles, the framework must satisfy a number of requirements. First, the framework must be able to extract information from articles of a target MediaWiki application. This is so the system isn't limited to only Wikipedia and can target any MediaWiki instance. Next, the framework must be modular and allow various means to extract information from a MediaWiki instance. Next, article information extraction should be reasonably fast.

It must be possible for any single article to be abstracted into a vector of features for purposes of classification. Because it's expected that we will be working with a large number of Wikipedia articles, the required physical storage space of article meta data must be reasonably small. Furthermore, due to the experimental nature of machine learning, it must be made straight 
forward to programmatically create multiple feature vector representations for the same article. It must also be convenient to define a various types of article features for constructing feature vectors.

For building classifiers, the framework must be able to support pluggable supervised learning algorithms. This is necessary for the experimental nature of this thesis. This way various learning algorithms can be benchmarked. Finally, rapid testing and prototyping of article quality models is a necessary due to the experimental nature of this thesis. Therefore the framework must make it convenient for defining and using models of article quality. 


\section{Chapter 4}

\section{Implementation}

\subsection{System Architecture}

The approach of this quality assessment system is to define multiple quality models for different classes of articles. Given some arbitrary article, a machine learning approach is then used to predict what predefined article class the article belongs to. Depending on that prediction, the appropriate quality model is applied to the article. The motivation behind using multiple models of quality rather than one is the observation that articles often vary greatly by nature, with each nature having different standards of quality. For example, an article on "Thomas Edison" may be deemed very high quality due to the number of references, detail, and written prose. However, when using the same criteria on an article such as "List of mountains by elevation", the article may be deemed low quality, despite being having the correct elevation figures for every known mountain in the world.

A high level overview of the entire automatic Wikipedia quality analysis system is shown in Figure 4.1. A crawler first extracts raw data from Wikipedia or any MediaWiki website. This raw information then gets processed into a state suitable for analysis. Next, this processed information is used to construct vectors of features. Each vector is associated with exactly one article. Finally, each feature vector is used as input to a battery of classifiers, which 


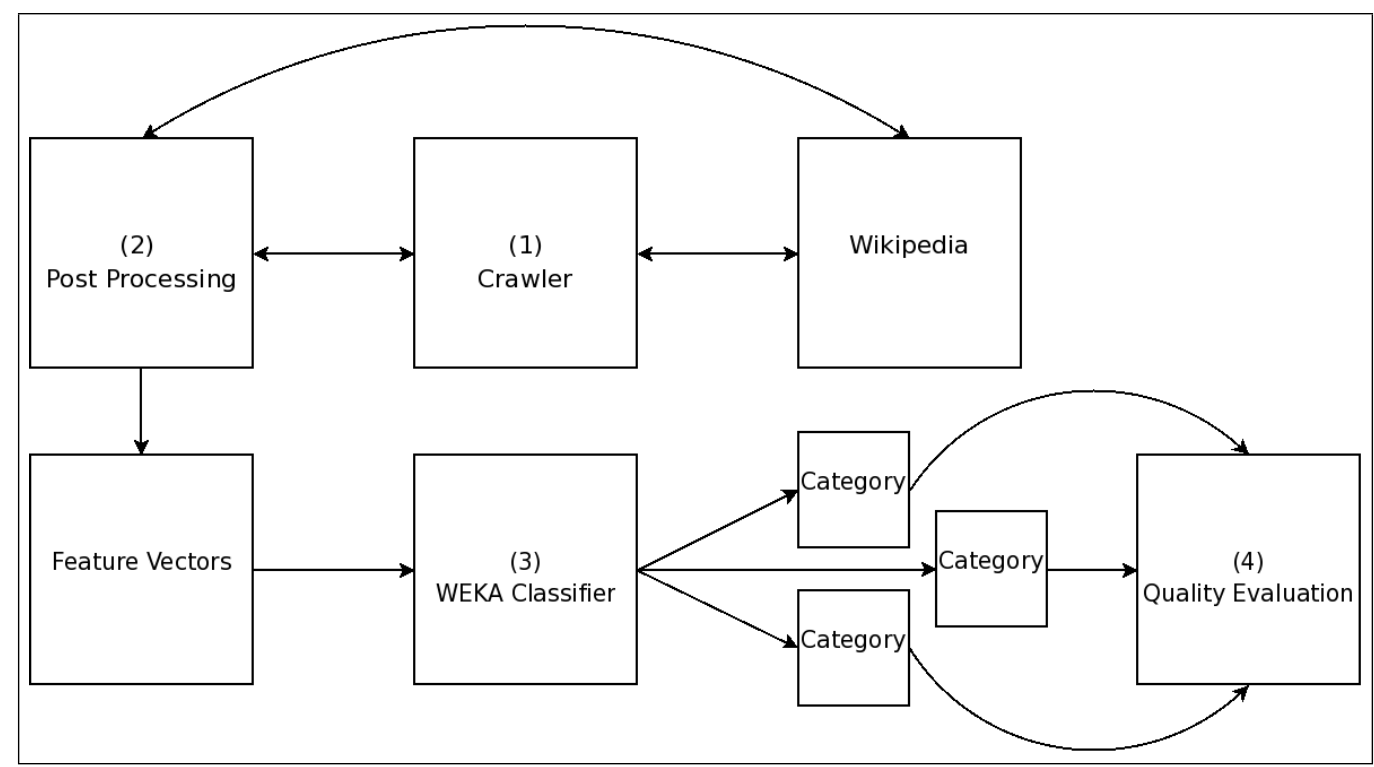

Figure 4.1: System Architecture

make a prediction on what type of class the article belongs to. Finally, depending on the outcome of the classification process, a specific model for quality is used to measure article quality.

\subsection{Wikipedia Crawler Foundation Classes}

The overall system architecture shown in Figure 4.1 is achieved through the Wikipedia Crawler foundation classes. The purpose of the foundation classes is to provide all of the functionality needed to gather information from any MediaWiki based website, and for interpreting that data into quality. The foundation classes are organized into seven major top level packages: articlefeature, classify, dataset, ir, qualitymodel, and crawler. Short descriptions of these top-level packages are shown in Table 4.2. 


\begin{tabular}{|l|l|}
\hline Name & Description \\
\hline articlefeature & $\begin{array}{l}\text { Extractable features from MediaWiki } \\
\text { articles }\end{array}$ \\
\hline classify & Wrapper for WEKA functionality \\
\hline dataset & $\begin{array}{l}\text { Representations for a collection of Me- } \\
\text { diaWiki articles }\end{array}$ \\
\hline ir & $\begin{array}{l}\text { Information retrieval related function- } \\
\text { ality }\end{array}$ \\
\hline qualitymodel & $\begin{array}{l}\text { Models for the quality of Wikipedia ar- } \\
\text { ticles }\end{array}$ \\
\hline crawler & $\begin{array}{l}\text { Functionality for retrieving raw infor- } \\
\text { mation from a MediaWiki application }\end{array}$ \\
\hline
\end{tabular}

Table 4.1: Top level packages

As shown in Figure 4.1, the first two steps for assessing the quality of an article is to collect article and post-process article information. Both of these steps are the tasks for the Wikipedia crawler. The Wikipedia crawler is assigned the task of extracting article information from Wikipedia or any MediaWiki website. The output of the Wikipedia crawler is a collection of RawPageInfo instances.

RawPageInfo objects are the core of the Wikipedia foundation classes. This class provides a high level representation of any given article from a MediaWiki web application. Instances of RawPageInfo are produced from Crawler objects. The main purpose of a Crawler is to collect information about Wikipedia articles and generate an associated RawPageInfo representation of those articles. There are numerous means to collecting article information, and thus there are numerous types of Wikipedia Crawlers. For instance, MediaWikiApiCrawler makes use of the PHP API provided by MediaWiki to collect information on articles. Likewise, SednaCrawler collects article information by querying a Sedna native XML database. Currently, the only available Crawler is the MediaWiki PHP API crawler.

Each Crawler may choose to cache RawPageInfo instances in a local database 
management system. Upon the creation of a RawPageInfo instance, in addition to returning that instance to the caller, the Crawler can store the instance in a RawPageInfoDB. There are many different types of RawPageInfoDB objects as there are many types of database management software. For instance, MySQLRawPageInfoDB represents a MySQL database of RawPageInfo objects. Likewise, H2RawPageInfoDB represents a H2 embedded database of RawPageInfo objects. When a request for an article is processed by a Crawler, the Crawler first looks in a RawPageInfoDB for that article. If a local copy isn't found or if the local copy is "stale", then the Crawler creates a new RawPageInfo and inserts it into the RawPageInfoDB, along with returning the object back to the caller.

The next step in the architecture is to create feature vectors of articles. This is done with Feature and FeatureVector objects. Once we have a RawPageInfo representation of an article, we can then use Feature objects to create customized views of the RawPageInfo object. A Feature is an object that takes in as input a RawPageInfo and derives a single value. For instance, the LengthFeature outputs the length of a given RawPageInfo, while a RevisionCountFeature outputs the total number of revisions belonging to the RawPageInfo. A more complete list of features can be found in Section 4.2.5.

Collections of features can be grouped together into a FeatureVector. A FeatureVector allows for a convenient way to create an aggregated view of a specific article. For instance, suppose a application programmer were to investigate the structural qualities of a certain article, such as its length or number of links. The programmer would then define a "structural" view for an article, which is a FeatureVector containing LengthFeature, HeaderCountFeature, WordCountFeature, and TotalInternalLinksFeature. For managing views 
of a large number of RawPageInfo instances, the Dataset class is used. With the Dataset class the application programmer can automatically load lists of Wikipedia articles specified by name and set a feature view for those articles.

Finally, classification of articles is done with an ArticleClassifier object. This object has two modes of operation, training and evaluation. For the evaluation operation, the input of an ArticleClassifier is a collection of FeatureVectors, and the output is a predicted class for each input vector. For the training operation, a collection of labeled vectors is required as input. An ArticleClassifier object handles the details of training and classifying RawPageInfo instances. The feature view of the entire supplied Dataset is used as the set of features for a machine learning classifier. In addition, ArticleClassifier objects allow the user to use various pre-implemented classification algorithms or create custom classifiers with the WEKA [40] Java library.

\subsection{1 classify package}

This is a package of wrappers for the WEKA machine learning Java library. There are two different classes to simplify two different tasks accomplished with WEKA. The first is the Dataset class. The Dataset class is a collection of feature vectors, with one feature vector per article. All articles within the Dataset are assumed to have the exact same number and type of features. This assumption is enforced by supplying a "header" to the Dataset. A header is simply a FeatureVector instance. The header acts as a view into the dataset. Once a dataset is loaded from a file and a view specified, the application programmer may export the dataset into a ARFF format for further analysis with the WEKA explorer. Short descriptions of the top-level classes for this 
package are shown in Table 4.2.1.

\begin{tabular}{|l|l|}
\hline Name & Description \\
\hline ArticleClassifier & WEKA wrapper for building classifiers \\
\hline Dataset & A collection of MediaWiki articles \\
\hline
\end{tabular}

Table 4.2: Top-level classify classes

Next is the ArticleClassifier class. This class is a simple wrapper over WEKA to easily train and evaluate a WEKA-based classifier. An ArticleClassifier is constructed with two parameters: A Dataset instance described above, and a WEKA machine learning algorithm. WEKA provides a wide variety of machine learning algorithms shown in Table 4.2.1. Once an ArticleClassifier is constructed, the following actions may be performed on that instance: buildClassifier(), crossValidate(), and classify(). The buildClassifier() method builds a classifier using the specified machine learning algorithm with the specified Dataset as a training set. The crossValidate() method takes in an integer $\mathrm{k}$, and performs k-fold cross validation with the specified Dataset. Once cross-validation is complete, the getEvaluation() method may be invoked to get a WEKA evaluation object. Finally, the classify() method takes in a RawPageInfo instance, and classifies that instance with either a positive or negative. 


\begin{tabular}{|l|l|}
\hline Algorithm Name & Package \\
\hline Alternating Decision Tree [20] & Tree \\
\hline AODE [58] & Bayes \\
\hline AODE With Subsumption Resolution [66] & Bayes \\
\hline Bayesian Logistic Regressions [21] & Bayes \\
\hline Bayesian Network & Bayes \\
\hline Citation kNN [56] & Multi Instance \\
\hline Complement Naive Bayes [48] & Bayes \\
\hline Conjunctive Rule & Rule \\
\hline Majority Decision Table [30] & Rule \\
\hline Discriminative Multinomial Naive Bayes & Bayes \\
\hline Fuzzy Logic Reasoning [9] & Misc \\
\hline Functional Tree [31] & Tree \\
\hline Gaussian Process [34] & Function \\
\hline Hidden Naive Bayes [65] & Bayes \\
\hline HyperPipe & Misc \\
\hline Nearest Neighbor [8] & Lazy \\
\hline K-Nearest Neighbors [8] & Lazy \\
\hline ID3 [44] & Tree \\
\hline Isotonic Regression & Function \\
\hline C4.5 [45] & Tree \\
\hline RIPPER [17] & Rule \\
\hline K* [16] & Lazy \\
\hline Lazy Bayesian Rules Classifier [67] & Lazy \\
\hline Least Median Square [49] & Function \\
\hline Logistic Model Tree [51] & Tree \\
\hline Sequential Minimal Optimization [42] & Function \\
\hline
\end{tabular}

Table 4.3: List of WEKA Machine Learning Algorithms

\subsection{2 qualitymodel package}

This package contains an abstract class, QualityModel, for building your own quality models. In addition, there are three different pre-implemented models: StabilizedModel, BlumenstockModel, and EuclideanDistance. Each of these models are discussed in greater detail in Section 3.2. A short description of the top-level classes of this package is shown in Table 4.2.2. 


\begin{tabular}{|l|l|}
\hline Name & Description \\
\hline AbstractModel & Base class inherited by all models \\
\hline BlumenstockModel & Quality model based on length \\
\hline ControversialModel & Quality model for controversial articles \\
\hline EuclideanDistanceModel & $\begin{array}{l}\text { Quality model which uses Euclidean } \\
\text { distance from a featured article cluster }\end{array}$ \\
\hline ListModel & Quality model for list articles \\
\hline StabilizedModel & Quality model for stabilized articles \\
\hline StubModel & Quality model for stub articles \\
\hline
\end{tabular}

Table 4.4: Top-level qualitymodel classes

\subsection{3 ir package}

This package contains everything related to the field of information retrieval and it's application to the retrieval of data from MediaWiki applications. Information retrieval is the science of searching documents, information within documents, and document meta data. In the context of a MediaWiki application, a "document" is a single revision of an article. Thus an article on "quick sort" may actually contain a large number of documents in its revision history. Within this package, the Document class captures information retrieval's concept of a document. A short description of top-level classes for this package is shown in Table 4.2.3.

\begin{tabular}{|l|l|}
\hline Name & Description \\
\hline Document & $\begin{array}{l}\text { Bag of words representation of Medi- } \\
\text { aWiki article content }\end{array}$ \\
\hline StandardStemAnalyzer & $\begin{array}{l}\text { Document post-processing wrapping } \\
\text { using Lucene }\end{array}$ \\
\hline WikitextStripper & Wikitext syntax remover \\
\hline StopWords & $\begin{array}{l}\text { Stopword remover of MediaWiki article } \\
\text { content }\end{array}$ \\
\hline Stemmer & English word stemmer \\
\hline
\end{tabular}

Table 4.5: Top-level ir classes

The ir.preprocessing sub-package contains all of the necessary classes for preparing article revisions for a conversion to a Document representation. 
The currently available pre-processing actions are stemming, stopword removal, and Wikitext markup removal. The Stemmer class is responsible for stemming the content of an article's revision. This Stemmer class is actually a wrapper for an existing snowball stemming algorithm Java implementation [3]. The underlying snowball algorithm can stem words from multiple languages. Since the focus of this thesis is the English edition of Wikipedia, the Stemmer class makes use of the English stemmer.

Stopword removal is handled with the StopWords class. This class is simply a hash table of words which is initialized by a file containing all of the specified stop words. The stop word list used by this class is a list freely available on the Internet [13].

The WikitextStripper class is responsible for taking the raw text of an article revision, and removing any unnecessary wikitext markup. The removal of removing wikitext markup from revision content is an interesting problem in itself, simply because the content includes a mix of wikitext formatting and XML tags. Wikitext is a markup specification consisting of interspersed text and tags. Common tags in the Wikitext specification include double square brackets indicating internal links to other Wikitext pages. The key difficulty associated with working with Wikitext content is that at the time of writing, there is no full Wikitext parser.

While progress has been made in solving this problem, the available parsers are still incomplete and unsuitable for this project. Despite these issues, the need to derive features may still exist. To simplify things, the assumption is made that any features which are a function of article content will not perform any natural language processing. Specifically, an article's content is treated as a bag of words with all formatting stripped. With this assumption, a relaxed parser was developed to simply recognize Wikitext and XML formatting. 
Optionally, pre-processing of an article revision's content may be achieved with the help of the Lucene Java library [19]. The StandardStemAnalyzer class implements an analyzer for Lucene. The StandardStemAnalyzer converts all text into lower case, removes stop words, and stems terms.

\subsection{4 wikipediacrawler package}

The crawler package contains all of the logic necessary for extracting information from a MediaWiki web application. By design, the crawler package allows for various means of article information extraction. However, currently it's only possible to extract article information through an exported MediaWiki API. The MediaWikiApiCrawler implements this logic. Short descriptions of the top-level classes of this package can be found in Table 4.2.4.

\begin{tabular}{|l|l|}
\hline Name & Description \\
\hline Crawler & $\begin{array}{l}\text { Base class inherited by all crawler im- } \\
\text { plementations }\end{array}$ \\
\hline H2RawPageInfoDB & $\begin{array}{l}\text { A RawPageInfo repository using the } \\
\text { H2 backend }\end{array}$ \\
\hline MySQLRawPageInfoDB & $\begin{array}{l}\text { A RawPageInfo repository using the } \\
\text { MySQL backend }\end{array}$ \\
\hline RawPageInfo & $\begin{array}{l}\text { A high level representation of a Me- } \\
\text { diaWiki article's revision history and } \\
\text { meta-data }\end{array}$ \\
\hline RawPageInfoDB & $\begin{array}{l}\text { Base class inherited by all RawPageInfo } \\
\text { repositories. }\end{array}$ \\
\hline
\end{tabular}

Table 4.6: Top-level wikipediacrawler classes 
When using a Crawler to create a RawPageInfo representation of an article, by default only the meta data of a revision is collected. The revision meta data includes the username of the author (or IP address for anonymous revisions), the time stamp, and the total length in bytes. The application programmer may optionally specify the maximum amount of revisions to extract the entire content for. This action is optional because of potential impacts on retrieval time explained in the next section. By default, extraction of article revision content is disabled. When this option is enabled, as the crawler receives an article revision's content, it utilizes the preprocessing classes described in Section 4.2.3.

\section{wikipediacrawler.mediawiki package}

The wikipediacrawler contains the logic to extract information from articles through the use of the MediaWiki API. The MediaWiki API is implemented in PHP and allows for programmatic use of all of Wikipedia's functions, including but not limited to: logging in, logging out, retrieving article revision history, and retrieving article meta information. Short descriptions of each top-level class in this subpackage is shown in Table 4.2.4.

\begin{tabular}{|l|l|}
\hline Name & Description \\
\hline MediaWikiApiCrawler & A Crawler implementation for \\
Wikipedia which makes uses of \\
the MediaWiki API
\end{tabular}

Table 4.7: Top-level wikipediacrawler.mediawiki classes

The advantage of extracting article information through the use of the MediaWiki API is its flexibility. A application programmer can extract article information from any MediaWiki web application exporting the MediaWiki 
API, regardless of whether or not the target MediaWiki web application is owned by the application programmer. Other advantages of using the MediaWiki API is a reduced overhead of running a database management system. By querying a remote MediaWiki application rather than our own, we save physical space to maintain the application, as well as CPU cycles.

Finally, utilizing the MediaWiki API effectively "outsources" the necessary logic to extract certain features. For instance, one approach to implement an article length feature is to retrieve the textual content of an article and count the number of bytes. However, with the MediaWiki API it's possible to directly request an article's length. Similarly, instead of parsing an article's content for internal links, it's possible to request the links directly through the MediaWiki API. However, despite the fact that much of the work work for feature derivation can be out sourced to the MediaWiki API, for more complex features parsing of revision content may still be involved.

The disadvantage of utilizing the MediaWiki API for article extracting is the speed at which the extracting process takes place. In order to extract article information, there is the additional overhead of transmitting information over the TCP/IP protocol from the client to the server. Furthermore, the transmission speed is limited by any MediaWiki API bandwidth controls. For instance, currently Wikipedia limits the retrieval of article revisions to 500 per request for an anonymous user and 5000 for a confirmed bot user. Additionally, if an application programmer requests the entire content of an article's revision history, then the MediaWiki API only allows 50 revisions per request to an anonymous user and 500 for a bot user. Because of bandwidth control enforcement, retrieving the revision history of a large article may actually require multiple requests to the MediaWiki API. If a MediaWikiApiCrawler is to be used in conjunction with a graphical front end, then the speed at which 
article information can be extracted becomes important.

To mitigate the impacts of slow retrieval of RawPageInfo objects, a Crawler makes use of threads and local caching. When a request is made to retrieve RawPageInfo objects for a large group of Wikipedia articles, the MediaWikiApiCrawler uses a pool of threads to query the MediaWiki API in parallel. By default, MediaWikiApiCrawler uses a pool of ten threads, however this number can be changed by the application programmer. Each thread consumes information in the request buffer and outputs a RawPageInfo instance into the output buffer. Access to these buffers are synchronized to avoid any race conditions. In the MediaWikiApiCrawler, each thread is actually a QueryWorker. A QueryWorker contains all of the logic to generate appropriate MediaWiki API queries, and in the case of large articles, dynamically schedule a sequence of requests.

Note that parallelism only occurs at the Wikipedia article name level rather than the MediaWiki API query level. Each QueryWorker will compete for a new article to process within the request buffer. If the information extraction of an article requires multiple requests to the MediaWiki API, then those requests are handled consecutively by a single QueryWorker.

A RawPageInfo representation of an article may optionally be stored in a local database management system. This is done by invoking the retrieve() method of a Crawler instance. A retrieve invocation extracts information from an article in the same manner described above with the following addition. Before utilizing the MediaWiki API, a RawPageInfo representation of the target article is first searched for in a RawPageInfoDB instance. If a matching RawPageInfo is found which isn't outdated according to a timestamp, then simply return that instance. Else, perform the same MediaWiki API extraction process described earlier, and store the resulting RawPageInfo 
in a RawPageInfoDB with a current timestamp.

Each element in a RawPageInfoDB is a tuple with a timestamp and a blob containing a serialized RawPageInfo instance. Serialized objects rather than a collection of tables are used for flexibility. This decision was made due to the convenience of implementation. Making a change to the contents of a RawPageInfo would result in a sequence of relation updates to the RawPageInfoDB. However, with a serialized object scheme, a change in a RawPageInfo object only requires that the RawPageInfoDB be re-populated. A problem with storing serialized objects in a RDBS the large amount of physical space required by a serialized object.

To reduce the physical disk usage of a RawPageInfoDB, serialized RawPageInfo instances are gziped before insertion, and de-compressed on access to the RawPageInfoDB. Furthermore, when storing the revision history of a article, only the content deltas rather then the entire content is stored in a RawPageInfo. This is possible through the use of the Javaxdelta3 library [2]. Storing the deltas of each revision is effective because each revision is an incremental update to an article's content. This is intrinsic to a on-going body of written work. When a revision is made, only part of the content from the previous revision is modified. Storing the entire content of each revision for an article contains a large amount of redundant information. The drawback of storing deltas is the addition computation needed to reconstruct a specific revision from deltas.

Currently there are only two types of RawPageInfoDB objects available: MySQLRawPageInfoDB and H2RawPageInfoDB. MySqlRawPageInfoDB stores RawPageInfo instances in a MySQL DBMS backend. Meanwhile, H2RawPageInfoDB stores RawPageInfo instances in a H2 backend. MySQLRawPageInfoDB is suitable for a more robust storage of RawPageInfo instances. On the other hand, 
H2RawPageInfoDB is provided when the administration and setup of a DBMS isn't possible.

\subsection{5 articlefeature package}

This package contains classes to define various features of a Wikipedia article.

A feature of an article is a defined as a characteristic of an article, which is extracted from an article's textual content. For instance, the number of bytes of an article can be used to define a article "length" feature. All article features found in this package are subclasses of Feature. Short descriptions of each top-level feature is shown in Table 4.2.5 


\begin{tabular}{|l|l|}
\hline Name & Description \\
\hline CategoryCountFeature & Number of categories for an article \\
\hline CitationCountFeature & Number of citations for an article \\
\hline ControversialTemplateFeature & $\begin{array}{l}\text { Whether or not an articles possesses a } \\
\text { controversial template }\end{array}$ \\
\hline DisambiguationCategoryFeature & $\begin{array}{l}\text { Whether or not an articles is placed un- } \\
\text { der a disambiguation category }\end{array}$ \\
\hline DisambiguationTitleFeature & $\begin{array}{l}\text { Whether or not an article's name indi- } \\
\text { cates a disambiguation article }\end{array}$ \\
\hline HeaderCountFeature & $\begin{array}{l}\text { Number of header sections within an } \\
\text { article's latest revision }\end{array}$ \\
\hline ImageCountFeature & $\begin{array}{l}\text { Number of images in an article's latest } \\
\text { revision }\end{array}$ \\
\hline LengthFeature & $\begin{array}{l}\text { Length in bytes of an article's latest re- } \\
\text { vision }\end{array}$ \\
\hline RatioInternalLinksFeature & $\begin{array}{l}\text { Ratio of internal links to external links } \\
\text { in an article's latest revision }\end{array}$ \\
\hline RevisionCountFeature & $\begin{array}{l}\text { Number of revisions an an article's re- } \\
\text { vision history }\end{array}$ \\
\hline StubCategoryFeature & $\begin{array}{l}\text { Whether or not an article is placed in } \\
\text { a stub category }\end{array}$ \\
\hline Number of external links for latest re- \\
vision of an article
\end{tabular}

Table 4.8: Top level articlefeture classes

Currently the foundation classes contain various pre-implemented article features. Roughly, the features can be categorized into two categories: structural and historical. Structural features describe characteristics of an articles latest revision. These types of features only take into account any current information in the latest revision. Historical features are features which take into account any aspect of an article's revision history. Structural features 
tend to describe quality of the latest incarnation of an article, while historical features tend to describe any trust or reliability of a Wikipedia article.

Category Count. This feature is implemented in the CategoryCountFeature class. This feature determines the number of categories the target article belongs to. In the MediaWiki context, a category is an organizational construct for articles. For instance, an article about binary search may contain a "Computer Science" category within the main namespace. Other category namespaces include "Talk" for discussions about articles, "Wikipedia" for articles in and about Wikipedia, "User" for pages containing contributor user profiles, and "File" for description pages of any user uploaded content. This category count feature includes categories from all namespaces.

Citation Count. This feature measures the total number of citations used in a given article. In this context, a citation is any pointer within the article content referencing a note located at the end of an article. Note that pointers in an article's content isn't always necessarily a citation in the context of article writing. At times, a pointer might simply indicate an editor's note clarifying some concept. Therefore, this feature might not be completely accurate.

Controversial Template. This feature determines if an article contains a template indicating controversial nature. In MediaWiki, a template is a section of an article consisting of a box with informative text. The background color of the template box reflects the nature of the template. Meanwhile, the text within the template box describes the template. MediaWiki uses these tem- 
plates to provide messages, warnings, or notifications unrelated to the content of an article to would be readers of an article. This particular feature checks if the target article contains the following templates: Template:Controversial, Template:Blpdispute, Template:Need-Consensus, and Template:Controversialissues. If any of these templates are found, then a true is returned, false otherwise.

Disambiguation Category. This features determines if the target article belongs to a "disambiguation" category. Within Wikipedia, it's common practice to give articles whose only purpose is to further help a reader disambiguate a search query, a disambiguation category. The disambiguation category used for such articles is commonly some kind of variation of "Disambiguation Pages". Thus, this feature verifies if any of the categories belonging to the target article has a name containing the phrase "disambiguation page". If this phrase is found, then the feature returns true. Otherwise, this feature returns false.

Disambiguation Title. When given a target article, this feature determines if the target article has the phrase "(disambiguation)" in its canonical title. If this phrase is found, then the feature returns true, otherwise the feature returns false. A disambiguation article in Wikipedia may or may not actually contain a disambiguation category, and usually has the phrase "(disambiguation)" in its title. This is because the maintenance of articles is voluntary, and its natural that some articles may not contain the proper categories or labels in the title. The purpose of this feature is to test for another "indicator" of disambiguation page. 
Header Count. This feature returns a numeric value representing the total number of headers found in the target article's content of the latest revision. A header is a structural component of an article which is typically used to indicate the start of a new section in the article. There are five different levels of headers supported by Wikipedia, with each level having different emphasis.

Image Count. A simple feature which returns a numerical value representing the total number of images found within the target article. Note that an image doesn't necessarily act as a complement to the current article's content. For instance, an image of a certain type of cat within the "Cat" article. An image within an article may only function as something cosmetic such as a navigation icon.

Article Length. With this simple feature, the length in total bytes of the latest revision of the target article is returned. This total length may take into account additional bytes due to Wikitext markup.

Internal Links to External Links. This is a simple feature which returns the ratio of internal links within an article to the number of external links within the article.

Revision Count. This is a simple feature which returns the total number of revisions in an article's revision history. 
Stub Category. A feature similar to the "disambiguation category" feature. However, instead this feature determines if a target article contains a category ending with the phrase "stubs". Within Wikipedia, articles which are incomplete are usually placed under a category indicating a stub. For instance, an unfinished article on a quick sort algorithm article may contain a category called "Computer Science Stubs".

Total External Links. This feature returns the total number of external links found within the target article's latest revision content. Within MediaWiki, a external link is type of hyperlink which points to a URL outside of the MediaWiki application. Common real world examples of external links found in Wikipedia articles are links to PDF files used as references.

Total Internal Links. This feature returns the total number of internal links found within the target article's latest revision content. Within MediaWiki, a internal link is a type of hyperlink which points to another article within the same MediaWiki application. For example, an article on "sorting algorithms" would contain numerous internal links to other specific sorting algorithm articles found within the same namespace.

Total Unique Editors. This feature iterates through a target article's revision history, and counts the number of different authors of the entire revision history. A author may be "validated"; a user which has resisted an account, or anonymous; a user which is only identifiable by IP address. 
Word Count. A simple feature which calculates the total number of words found in the latest revision of the target article. This feature makes uses of the ANTLR parser class and requires the input RawPageInfo representation to contain full text content of the revision history.

\section{Normalized Density Features}

This family of features output the "normalized density" of some characteristic of the target article. Normalized density features exists for: total internal links, total external links, image count, header count, and citation count. Normalized density of a function $\mathrm{f}$ is the value of $\mathrm{f}(\mathrm{x})$ divided by the total length in bytes of the article. Both the value of $f(x)$ and article length are further normalized taking its logarithm.

Normalized density features are useful for quality assessment of Wikipedia articles. For instance, any two high quality articles in Wikipedia may not have the same number of images or headers, thus a measurement relying on the absolute number of images or headers is not useful. The difference between these two articles may be because of differences in article size. Normalized density features to to address this problem.

\section{PageRank Analysis of Wikipedia Snapshot}

In 1995, PageRank was developed by Larry Page and Sergey Brin at Stanford University [41]. To develop a new search engine for the World Wide Web, Page and Brin needed a procedure for determining the importance of Web pages through link analysis. By 1998, Page and Brin eventually produced a search engine functional prototype name Google, with PageRank as its basis.

The PageRank algorithm is used for link analysis of pages in the World 
Wide Web. In this algorithm, weights are assigned to web pages. A web pages weight then contributes weight to any other web pages the original web page links to. Thus, the "importance" of any web page is proportional to the of any other pages linked to the original page.

It isn't too farfetched to think of articles in a MediaWiki application as a network of "Web pages". A webpage and a MediaWiki document are very similar. Both have a means to link to other Web pages or articles. The differences is that a MediaWiki articles has two different types of links: internal and external links. A internal link is a link which points to another article within the same MediaWiki application. On the other hand, an external link is a link which points to some location outside of the MediaWiki application. We can thus apply PageRank analysis by focusing on internal inks and ignoring the external links of MediaWiki articles.

When performing link analysis of articles from Wikipedia using PageRank, it's necessary to have a entire copy of all of Wikipedia's articles. For this purpose a snapshot of the entire Wikipedia content from 5-20-2009 was acquired. This snapshot contains over 20 gigabytes of information on over 8 million articles. Much of this physical space is due to the actual content of each article. Note that this snapshot only contains article content for the latest (at the time) revision. When performing PageRank analysis, an article's complete revision history isn't necessary.

The PageRank algorithm requires multiple iterations over the entire set of "vertices" within the network of interconnected articles in the English edition of Wikipedia. It would be ideal to hold all vertices and edges of this network into main system memory for performance reasons. Unfortunately, the 8 million article count of the English snapshot is too huge to fit into 2 Gigabytes of main system memory. However, If we make certain assumptions about the 
snapshot, then the total number of articles within the snapshot can be reduced drastically.

The first assumption is that articles within the "main" namespace contain the interesting articles, while articles from the remaining namespaces are irrelevant and can thus be pruned from the snapshot. Within MediaWiki there are 11 different namespaces for articles. The first is the "main" namespace which contains all encyclopedia articles, lists, disambiguation pages, and redirects. The "user" namespace contains pages created by user of the MediaWiki application for their own personal use such as user profiles. Next, is the "Wikipedia" namespace, which includes any pages related to Wikipedia itself such as articles on editing policies and processes. The "File" namespace includes pages describing any upload files such as images or audio. The "MediaWiki" template contains interface texts which appear automatically on generated pages. The "Template" namespace includes any page containing templates which can be included into any article. The "Help" namespace includes any pages which provide assistance to users regarding the use of Wikipedia and related software. The "Category" namespace includes articles for specific categories which lists other articles under that category and any sub-categories. Finally, the "Portal" namespace contains articles which help readers find a certain subject of interest.

The next assumption is that redirect pages within the "main" namespace do not contain any back links and thus serve no purpose for PageRank link analysis and can thus be pruned from the snapshot. In Wikipedia, a redirect article is an article whose only purpose is to automatically upon loading redirect to the appropriate page. Redirect pages are commonly used as a type of "auto correction" for typos of article names entered by users. In the actual snapshot, there might be instances of non-redirect articles linking to redirect 
pages. This is possible because the structure of the Wikipedia social network is constantly evolving. A redirect page may be an important link to some other article, which may greatly affect the final PageRank value of an article after multiple iterations of the algorithm.

After using these two assumptions to prune the English Wikipedia snapshot, only 3 million articles remain, which can easily be fit into 2 gigabytes of memory. To perform the algorithm, 32-bit integers weren't sufficient. Instead BigDecimal objects from the Java SDK were used for intermediate PageRank calculations. The downside to this is a performance hit when performing a PageRank iteration. With over 3 million articles held in main memory, 50 page rank iterations were performed and the result PageRank values for each article were saved to a database.

The PageRank algorithm assigns a probability to an article. This probability is difficult to interpret when there are over 3 million articles. To interpret these PageRank probabilities, the articles within the snapshot are ranked using a percentile like ranking based on their PageRank probabilities. Each page is ranked from 0 to 100. This ranking value for some arbitrary article A indicates what percentage of the articles within the snapshot have a PageRank value equal to or less than article A. The higher the rank, the higher PageRank the article has. For example, an article with a rank of 50 indicates half of the articles within the dataset have a equal or lower PageRank. The process of assigning these relative ranks is done by sorting each article within the snapshot by PageRank probability, and then determining the final rank for each article based on that article's position in the sorted list. 


\section{Chapter 5}

\section{Validation}

Prior research on information quality in Wikipedia, described in Section 2.3 $([25,33,64,47,24])$ approaches computing quality of an article in a uniform manner: for each proposed method the quality of any and all articles is estimated in exactly the same way. In contrast, our approach is to recognize that there may be inherent differences in how the quality of different Wikipedia articles should be estimated. We use different techniques and/or information for articles which belong to different "categories."

Our pilot study was designed to test the hypothesis, that using separate models to compute quality estimates for articles of different types leads to higher accuracy. We selected two categories of articles: stabilized (see Section 3.2.1) and controversial (see Section 3.2.2) As the means of validation, we elected to compare the predictions of our models to the opinions of casual Wikipedia users. As such, the study described below pursued two main questions: (1) do information quality models for stabilized and controversial articles adequately predict human opinion of the stabilized and controversial articles respectively? and (2) does using two models to predict information quality lead to more accurate predictions, then using a single information quality model for all articles? The following hypotheses are proposed:

- $H 0_{0}$ : There is no difference in article quality evaluation between the 
stabilized and controversial models.

- H0: There is a difference in article quality evaluation between the stabilized and controversial models.

- $H 1_{0}$ : The type/category of an article does not matter when evaluating its quality.

- H1: The type/category of an article matters when evaluating its quality.

- $H 2_{0}$ : Our stabilized model does not approximate a stabilized article's quality as determined by humans better than the controversial model.

- H2: Our stabilized model approximates a stabilized article's quality as determined by humans better than the controversial model.

- $H 3_{0}$ : Our controversial model does not approximates a controversial article's quality as determined by humans better than the stabilized model.

- H3: Our controversial model approximates a controversial article's quality as determined by humans better than the stabilized model.

\subsection{Experimental Setup}

To test our information quality models we conducted an experimental study in which participants read a variety of Wikipedia pages and ranked their information quality. The study involved 247 Cal Poly students who were enrolled

during the Fall 2009 quarter in an array of courses (both major courses and service courses) offered by the Computer Science department.

To conduct the study, we have created a dataset consisting of 100 Wikipedia articles and used the versions of those articles offered to the readers on October 
20, 2009. We used the "frozen" version of each article instead of the current version to ensure that all subjects who observed/read a specific Wikipedia article in our study accessed exactly the same content. Among the 100 articles, 51 were selected by us while the remainder of the articles were chosen randomly, using Wikipedia's "return a random article" feature. We chose to select a subset of articles directly to ensure that articles of each category we were interested in were present in the dataset. We also chose some articles to ensure the presence in the dataset of articles about topics that are both wellknown to study participants (e.g., "Cal Poly") as well as rather unknown (e.g., "Choi Jai-Soo"). A complete list of selected articles for this study is found in Appendix A. We applied stabilized and controversial articles classifiers obtained from using WEKA's [40] SMO algorithm [42, 28] implementation. Table 5.1 shows that of our 100 articles, 50 were classified as stabilized, 29 as controversial, 10 as both, and 31 as neither.

\begin{tabular}{|l|c|}
\hline Article Type & Total \\
\hline All & 100 \\
\hline Stabilized & 50 \\
\hline Controversial & 29 \\
\hline Both & 10 \\
\hline Neither & 31 \\
\hline
\end{tabular}

Table 5.1: Survey Article Classification

Each study participant, via a specially designed on-line software tool (see Figure 5.2) received access to eight articles from our sample. We decided to limit the expected time the participants spend on the survey to no more than one hour. We conducted a number of pre-survey trial runs and established that it took a person about 50-60 minutes to complete the survey when provided with eight Wikipedia articles randomly selected from our dataset. The survey software maintained information on the number of times each article has been 
assigned to study participants. When a new user accessed the software, a list of eight different articles was randomly drawn from our dataset, with the probability distribution which granted article(s) with the fewest number of assignments the highest chance of being selected. Use of this procedure lead to each article being shown to roughly the same number of participants. In our study, each article was viewed and assessed by 18-20 participants.

For each page, we asked the participant to (a) read it, (b) evaluate its information quality and (c) specify the level of familiarity with the topic of the article. Participants could evaluate the information quality on a scale from 1 to 5 with mid-points (e.g. 2.5, 3.5) between integer scores allowed. The familiarity was evaluated on a scale from 1 to 3 with allowed mid points. The full scales are shown in Tables 5.2 and 5.3

\begin{tabular}{|l|l|}
\hline Value & Description \\
\hline 1.0 & Fail. Not ready for public consumption \\
\hline 1.5 & - \\
\hline 2.0 & Poor. Requires more revisions \\
\hline 2.5 & - \\
\hline 3.0 & Average. Serves its purpose \\
\hline 3.5 & - \\
\hline 4.0 & Good. Nearly complete \\
\hline 4.5 & - \\
\hline 5.0 & Great. Requires minor/no further revisions \\
\hline
\end{tabular}

Table 5.2: Survey Article Quality Scale

\begin{tabular}{|l|l|}
\hline Value & Description \\
\hline 1.0 & The subject is new to me \\
\hline 1.5 & - \\
\hline 2.0 & I have decent understanding \\
\hline 2.5 & - \\
\hline 3.0 & I'm an expert at the subject \\
\hline
\end{tabular}

Table 5.3: Survey Confidence Scale

Before taking the survey, the participants created accounts and logged in 
(see figure 5.1). When participants created accounts, they provided their real first name and last name. The first and last name was required for grading purposes and was provided to the course instructors to allow them to assess extra credit in the course. This information wasn't used in the study itself. When the participant finished account creation, he/she was provided with a random but unique userID to login.

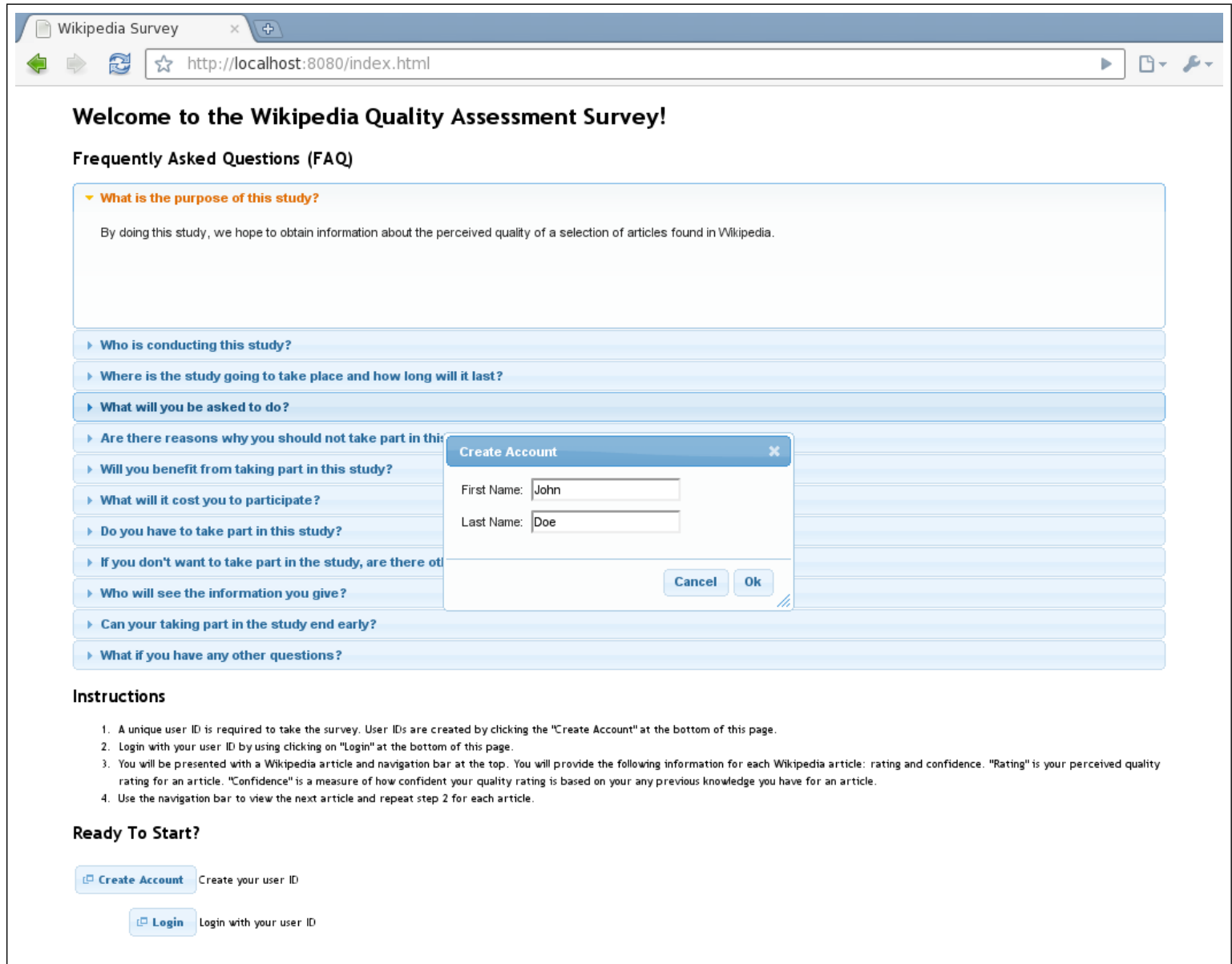

Figure 5.1: Survey Account Creation

As participants logged in for the first time, the survey software selected a number of Wikipedia articles from a preselected pool for each participant. Each participant in a single experiment received the same number of articles (in our case, 8). The number of articles in the pool was determined to be 
100. The survey software choose the articles in such a way as to evenly assign all articles within the pool among all volunteers (see Figure 5.2). The main survey screen contained a survey bar located on top and a main content pane displaying a selected Wikipedia article. The participant were asked to read the presented article. After reading each article, the participant used the survey bar to submit a quality assessment and his/her expertise level for each assigned article.

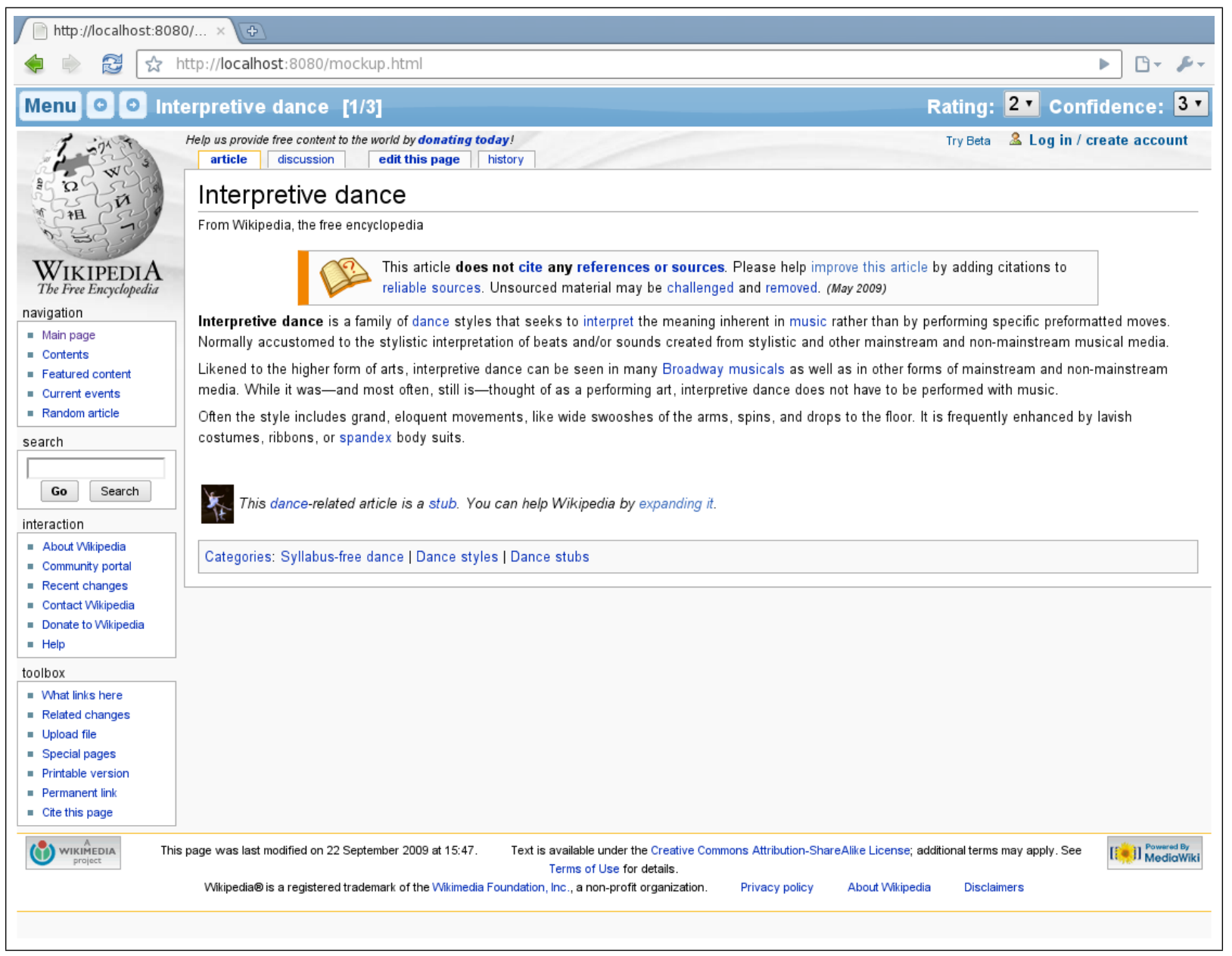

Figure 5.2: Main Survey Screen

On the survey bar, the participant selected Next or Back to navigate the list of survey articles. The survey remembered all of the participants' answers for each article such that work wasn't lost as the volunteer navigated the list 
of survey articles. Furthermore, the participant was free to abort the survey and resume at any time by logging out and in.

A list of surveyed articles and final student results of can be found in Appendix A. These results form the basis for our described hypotheses.

\subsubsection{Measures}

At the conclusion of the survey, we had accumulated a number of information quality and user confidence ratings for each article $A$ provided by individual participants. We used the average user rating $\overline{q_{u}}(A)$ to aggregate user opinion about each article. For our pilot study, we considered 69 of the 100 articles which were classified as stabilized or controversial in the analysis described below. For each article $A$ from this list, we computed two scores $q_{s}(A)$ and $q_{c}(A)$ using the stabilized and controversial information quality models described in Section 3.2 respectively, and the score $q_{m i x}(A)$, which was computed as follows:

$$
q_{m i x}(A)=\left\{\begin{array}{cc}
q_{s}(A) & \text { A is stabilized, not controversial; } \\
q_{c}(A) & \text { A is controversial, not stabilized; } \\
\frac{q_{s}(A)+q_{c}(A)}{2} & \text { A is stabilized and controversial. }
\end{array}\right.
$$

Further, we computed the errors of prediction $\delta_{s}(A)=q_{s}(A)-\overline{q_{u}}(A)$ and $\delta_{c}(A)=q_{c}(A)-\overline{q_{u}}(A)$ and $\delta_{m i x}=q_{m i x}(A)-\overline{q_{u}}$ for the stabilized, controversial models and mixed models respectively. To test our hypotheses, we compared

the average overall prediction errors for each model: $\bar{\delta}_{c}=\frac{1}{|S|} \sum_{A \in S} \delta_{c}(A)$, $\bar{\delta}_{s}=\frac{1}{|S|} \sum_{A \in S} \delta_{s}(A)$ and $\bar{\delta}_{m i x}=\frac{1}{|S|} \sum_{A \in S} \delta_{m i x}(A)$ (here, $S$ is a set of articles over which the prediction error is computed). 


\subsubsection{Hypothesis $H 0_{0}$ and $H 0$}

For this hypothesis we are interested in the relationship between quality as reported by our stabilized and controversial models. Each of the 100 survey articles is evaluated with the stabilized and controversial models described in Section 3.2. The probability distribution of these two models is then compared using Student t-test. A resulting test statistic that satisfies a 5\% significance level rejects hypothesis $H 0_{0}$.

\subsubsection{Hypothesis $H 2_{0}, H 2, H 3_{0}$, and $H 3$}

We are interested in the amount of error our stabilized and controversial models produce on articles classified as stabilized articles and those classified as nonstabilized. Error in this case is the absolute value of the difference between an article's quality as reported by humans and the measure outputted by the stabilized or controversial model. Because the survey article quality scale of 1 to 5 differs from the quality scale of 0 to 1 of the stabilized model, each article quality score of the survey results is converted to a 0 to 1 scale accordingly. This conversion process is done before calculating the deltas.

The deltas for stabilized and controversial models are then collected for all articles classified as stabilized and all articles classified as non-stabilized. This produces two sets of results, one for articles classified as stabilized and one for articles classified as controversial. For each set of results, Student t-test is used between the stabilized and controversial deltas. Resulting test statistics that satisfies a $5 \%$ significance level rejects hypothesis $H 2_{0}$ and $H 3_{0}$. 


\subsubsection{Hypothesis $H 1_{0}$ and $H 1$}

Unfortunately, our main hypothesis $H 1$ is a qualitative one. The results of the tests performed for the remaining hypotheses will provide evidence for the acceptance or rejection of hypothesis $H 1$. In addition, another experiment based on rank order is conducted. To perform this experiment, the survey articles are divided into a list of articles classified as stabilized and another classified as controversial. Each list is then assigned three different ranking orders: survey ratings, stabilized score, and controversial score. A rank is a numerical value, where the lowest rank represents the lowest quality rating and the highest rank represents the highest quality rating. Every pair between survey articles is then compared. A pair of articles where the relative comparison of survey rating rankings doesn't match the relative comparison of stabilized/controversial rankings indicates a ranking error by our quality models. The percentage of errors over all possible pairs is then reported for stabilized and controversial models.

\subsubsection{Miscellaneous}

From the survey data, other interesting plots and statistics are composed and presented. This includes a scatter plot of average student rating vs article PageRank, and T-test results involving our quality models for stub and list article categories.

\subsection{Results}

Figure 5.4 depicts the results of the stabilized method prediction, i.e., $q_{s}$, (Section 3.2.1) on articles classified as stabilized plotted vs. the average reader 
opinion $\bar{q}_{u}$. Figure 5.5 shows $q_{c}$, the controversial scores, plotted vs. the average reader opinion $\bar{q}_{u}$ for articles classified as controversial. Table 5.6 shows the $\bar{\delta}_{s}, \bar{\delta}_{c}$ and $\bar{\delta}_{m i x}$ for the set of stabilized articles, the set of controversial articles and the set consisting of both stabilized and controversial articles.

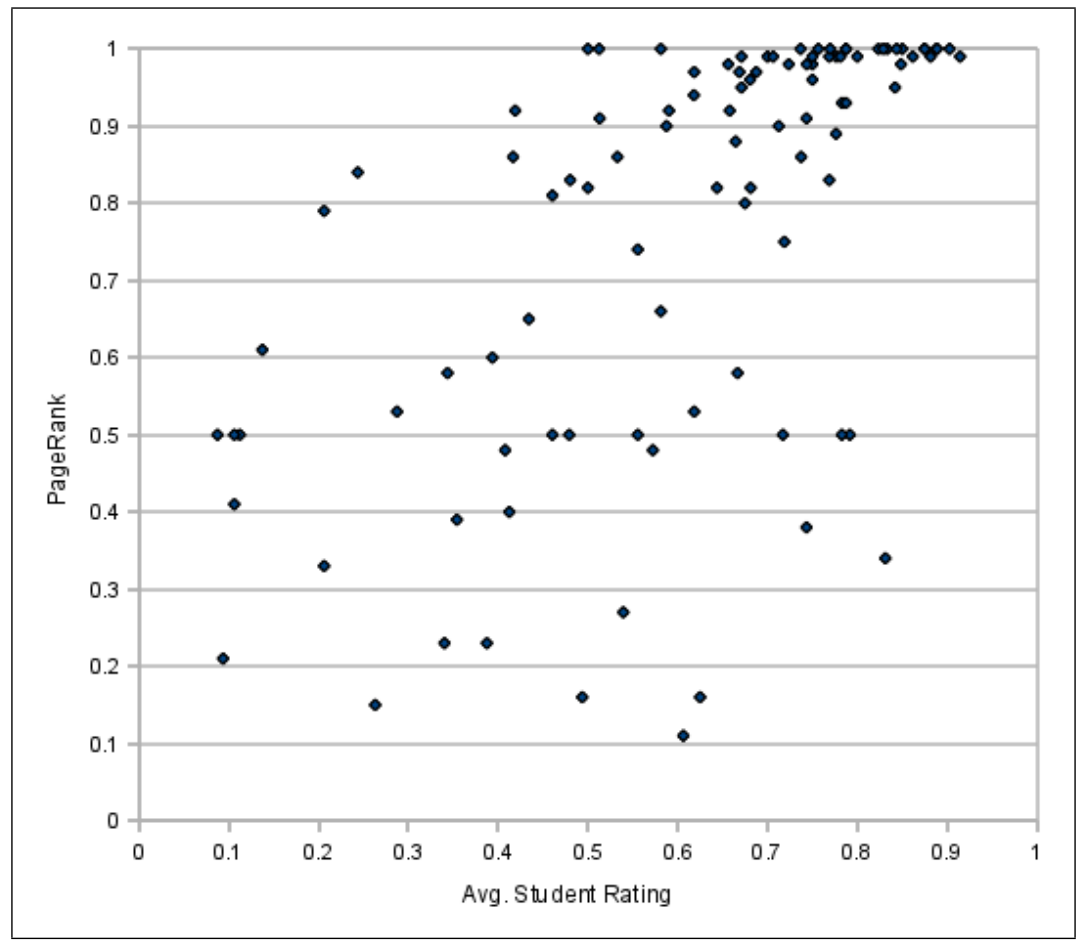

Figure 5.3: Avg Rating vs PageRank 


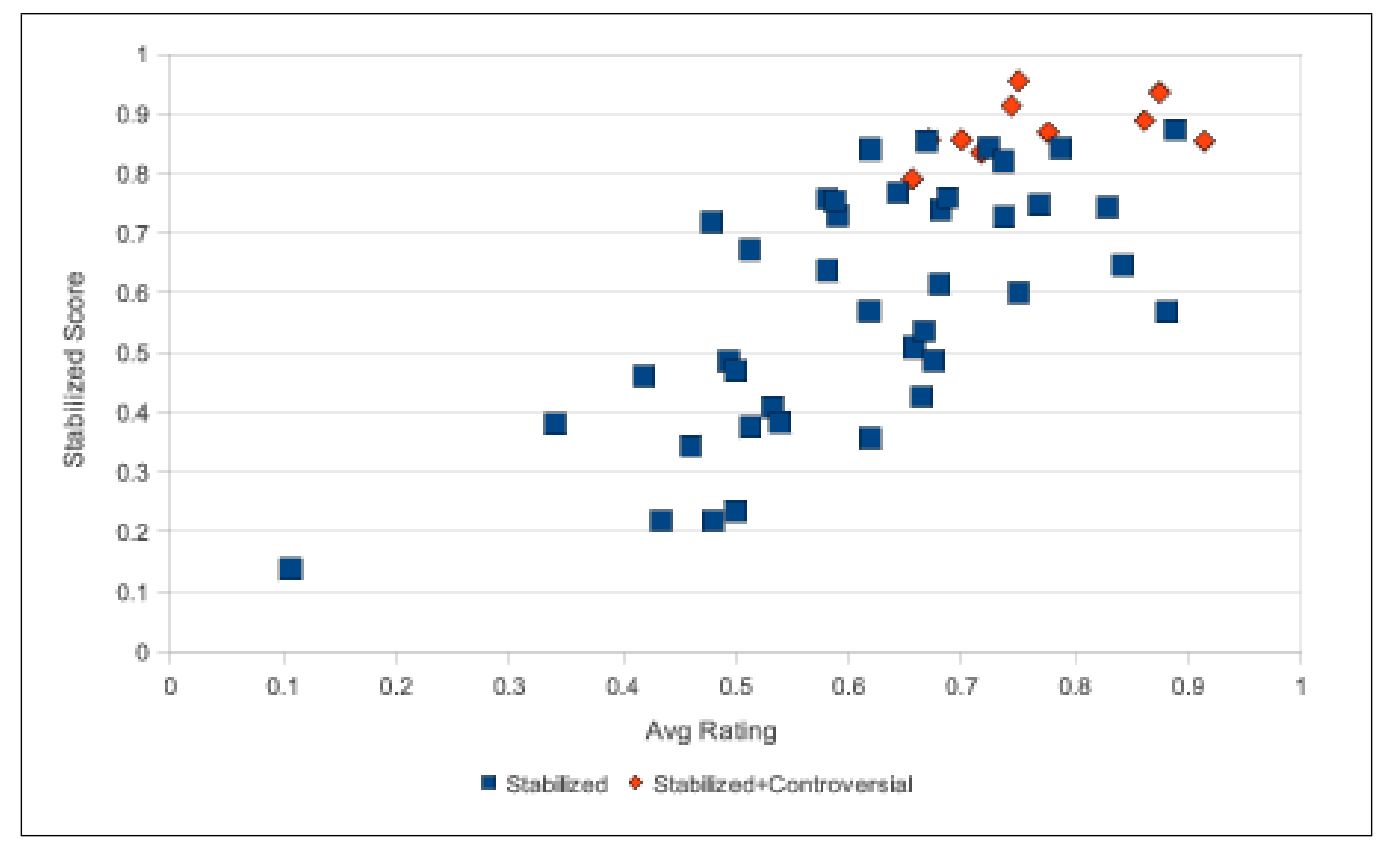

Figure 5.4: Avg Rating vs Stabilized Score

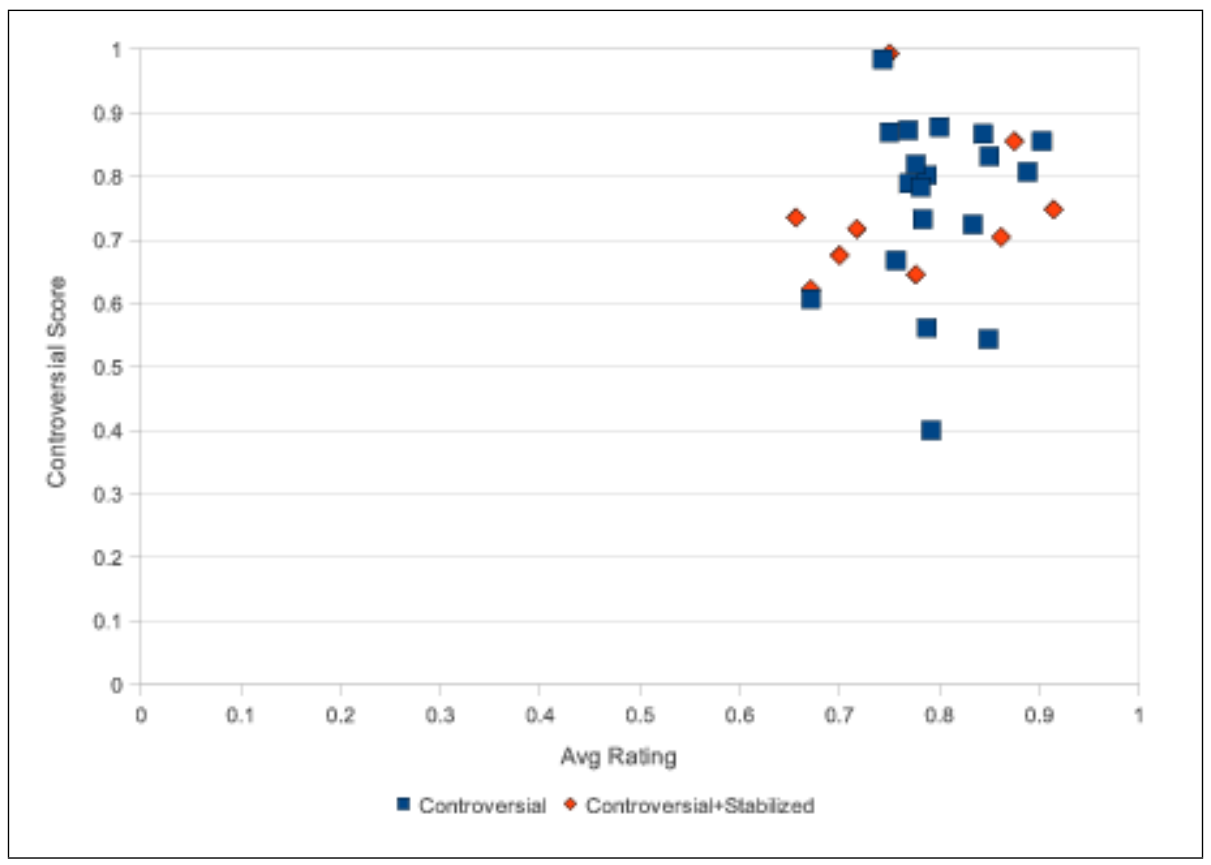

Figure 5.5: Avg Rating vs Controversial Score

\begin{tabular}{|l|l|l|l|l|}
\hline & N & Mean & StDev & SE Mean \\
\hline Stabilized Scores & 100 & 0.599 & 0.2675 & 0.027 \\
\hline Controversial Scores & 100 & 0.765 & 0.138 & 0.014 \\
\hline
\end{tabular}

Table 5.4: H0: Descriptive statistics 


\begin{tabular}{|l|}
\hline DF: 148 \\
\hline T-Value: -5.515 \\
\hline Stabilized Scores $\neq$ Controversial Scores: P-Value $=<.00001$ \\
\hline
\end{tabular}

Table 5.5: H0: T-Test Results

\begin{tabular}{|l|c|l|l|l|}
\hline & N & $\begin{array}{l}\text { Stabilized } \\
\text { Model }\end{array}$ & $\begin{array}{l}\text { Controversial } \\
\text { Model }\end{array}$ & Mixed Model \\
\hline Stabilized Articles & 50 & $\begin{array}{l}\text { Mean: } 0.127 \\
\text { StDev: } 0.0781\end{array}$ & $\begin{array}{l}\text { Mean: 0.201 } \\
\text { StDev: 0.145 }\end{array}$ & $\begin{array}{l}\text { Mean: } 0.119 \\
\text { StDev: } 0.0802\end{array}$ \\
\hline $\begin{array}{l}\text { Controversial Arti- } \\
\text { cles }\end{array}$ & 29 & Mean: 0.124 & Mean: 0.103 & Mean: 0.0979 \\
\hline Both Categories & 69 & $\begin{array}{l}\text { Mean: } 0.127 \\
\text { StDev: } 0.811\end{array}$ & $\begin{array}{l}\text { Mean: } 0.175 \\
\text { StDev: 0.141 }\end{array}$ & $\begin{array}{l}\text { Mean: } 0.116 \\
\text { StDev: } 0.0880\end{array}$ \\
\hline
\end{tabular}

Table 5.6: Mean absolute prediction errors

\begin{tabular}{|l|}
\hline \hline Error: Mix vs. Stabilized (all articles) \\
\hline \hline DF: 135 \\
\hline T-Value: -0.7639 \\
\hline Mix $<$ Stabilized: P-Value $=<0.2231$ \\
\hline \hline Error: Mix vs. Controv. (all articles) \\
\hline \hline DF: 113 \\
\hline T-Value: -2.95 \\
\hline Mix $<$ Controv.: P-Value $=<0.0019$ \\
\hline Error: Mix vs. List (all articles) \\
\hline \hline DF: 195 \\
\hline T-Value: -4.5399 \\
\hline Mix $<$ List: P-Value $=<0.00001$ \\
\hline \hline Error: Mix vs. Stub (all articles) \\
\hline \hline DF: 185 \\
\hline T-Value: -10.5028 \\
\hline Mix $<$ Stub: P-Value $=<0.00001$ \\
\hline \hline
\end{tabular}

Table 5.7: Mixed vs. stabilized, controversial, stub, and list models 


\begin{tabular}{|l|}
\hline Error: Stub vs List (Stub Articles) \\
\hline \hline DF: 31 \\
\hline T-Value: -1.7073 \\
\hline Stub $<$ List: P-Value $=<0.0489$ \\
\hline \hline Error: Stub vs Stabilized (Stub Articles) \\
\hline \hline DF: 39 \\
\hline T-Value: -1.5546 \\
\hline Stub $<$ Stabilized: P-Value $=<0.0641$ \\
\hline \hline Error: Stub vs Controv. (Stub Articles) \\
\hline \hline DF: 25 \\
\hline T-Value: -5.2419 \\
\hline Stub $<$ Controv.: P-Value $=<0.00001$ \\
\hline \hline Error: Stabilized vs Controv. (Stabilized Articles) \\
\hline \hline DF: 75 \\
\hline T-Value: -3.1897 \\
\hline Stabilized $<$ Controv.: P-Value $=<0.0010$ \\
\hline \hline Error: Stabilized vs List (Stabilized Articles) \\
\hline \hline DF: 88 \\
\hline T-Value: -4.3158 \\
\hline Stabilized $<$ List: P-Value $=<0.00001$ \\
\hline \hline Error: Stabilized vs Stub (Stabilized Articles) \\
\hline \hline DF: 85 \\
\hline T-Value: -10.7614 \\
\hline Stabilized $<$ Stub: P-Value $=<0.00001$ \\
\hline \hline Error: Controv. vs Stabilized $($ Controv. Articles) \\
\hline \hline DF: 52 \\
\hline T-Value: -0.8678 \\
\hline Controv. $<$ Stabilized: P-Value $=<0.1947$ \\
\hline \hline Error: Controv. vs List $($ Controv. Articles) \\
\hline \hline DF: 48 \\
\hline T-Value: -3.9091 \\
\hline Controv. $<$ Stabilized: P-Value $=<0.0001$ \\
\hline \hline Error: Controv. vs Stub (Controv. Articles) \\
\hline \hline DF: 45 \\
\hline T-Value: -15.8962 \\
\hline Controv. $<$ Stub: P-Value $=<0.00001$ \\
\hline \hline
\end{tabular}

Table 5.8: T-test results for models within their own articles 


\begin{tabular}{|l|l|l|}
\hline & Stabilized Score & Controversial Score \\
\hline Stabilized Articles & Error: 292 & Error: 584 \\
& Correct: 933 & Correct: 641 \\
& \% Error: 0.238 & \% Error: 0.527 \\
\hline Controversial Articles & Error: 214 & Error: 185 \\
& Correct: 192 & Correct: 221 \\
& \% Error: 0.527 & \% Error: 0.456 \\
\hline
\end{tabular}

Table 5.9: Stabilized \& Controversial Rank Error

\subsection{Analysis}

As shown in Figures 5.4 and 5.5, when applied to stabilized articles only, the stabilized model showed clear positive correlation with the average opinion. The correlation between the controversial model predictions and the reader opinion for controversial articles appears to be somewhat less pronounced (as seen on Figure 5.5), however excluding a few outliers, there is still a distinct positive correlation. In fact, Table 5.6 shows that the average error for controversial articles scored by the controversial model is 0.103 (with standard deviation of 0.0989): lower than 0.127, the average error for stabilized articles scored by the stabilized model (with standard deviation of 0.0781). Both methods achieve an error of $10-12 \%$, which, given the simplicity of the model suggests to us, that the methods are reasonably accurate.

As shown in Table 5.2, the $\mathrm{T}$ value between these two distributions is 5.515 with a two tailed $\mathrm{P}$-value of 0.00001 . This $\mathrm{T}$ value satisfies our $5 \%$ significance level. Thus, we reject hypothesis $H 0_{0}$ and accept $H 0$, concluding that stabilized and controversial models evaluate quality differently from each other.

In our second experiment, Table 5.6 shows that the mean absolute error of the stabilized model is lower than the mean absolute error of the controversial model for stabilized articles. Furthermore, as shown in Table 5.7 the T-value 
between these two distributions is -3.1987 with a one tailed P-value of 0.0010 . This T-value satisfies our 5\% significance level. Thus, we reject hypothesis $H 2_{0}$ and accept $H 2$, concluding that our stabilized model produces smaller error than our controversial model for stabilized articles.

On the other hand, the results in Tables 5.6 and 5.7 don't give us the ability to universally support hypothesis H3. As shown in Table 5.6, the mean absolute error of the stabilized model is larger than the controversial model error. However, in Table 5.7 the T-value between these distributions results in a one-tailed $\mathrm{P}$-value which doesn't satisfy our $5 \%$ level significance level. Therefore, we accept hypothesis $H 3_{0}$. Acceptance of $H 3_{0}$ indicates that our current model for controversial articles is far from perfect.

The results in Table 5.2 show a similar trend. In this table, the rank error of our stabilized model is smaller than the rank error of our controversial model for stabilized articles. However, in the case of controversial articles, our stabilized model had a larger rank error than our controversial model. In addition, our controversial model has a smaller rank error for controversial articles than stabilized articles. However, this improvement in rank error isn't as great as the mean absolute error shown in Table 5.6. The sum of these results provide strong evidence for our qualitative hypothesis $H 1$.

Finally, as shown in Table 5.6, the mixed scoring model outperforms the other two models (mean error of 0.116 vs. mean errors of 0.127 and 0.175 ). In Table 5.7, the Student T-Test shows that the difference between mean errors for the mixed and controversial models is statistically significant at the $5 \%$ significance level, and the difference for the mixed and stabilized models is not.

Out of curiosity, Figure 5.3 shows a plot of average student rating versus article PageRank. From this figure, there appears to be a correlation. It's 
observed that the articles highly rated by students had the highest PageRank. However, there are some outliers. On the bottom left portion are a group of low PageRank articles highly rated by the students. 


\section{Chapter 6}

\section{Conclusion and Future Work}

We presented the first in a series of results comparing a variety of quality models for Wikipedia articles with the opinions of casual Wikipedia readers. In this paper, we provided evidence for the key assumption behind our approach to measuring information quality: that quality of articles of different "type" should be computed using different means. We also introduced a new approach to validating models: validating quality estimates against the combined opinion of multiple casual Wikipedia readers, rather than against opinions of individual experts. Finally, we developed a framework for retrieving article information from a MediaWiki instance.

We plan to explore the work in [14] and this thesis further by studying the following issues:

Validation of Other Models. First, we are interested in validating other article quality models $[33,64,47,24]$ versus the casual reader opinion. This process involves implementing other quality models in software and running the same experiments conducted for this thesis. The existing data from this thesis can be re-used.

Article Category Expansion. Second, we plan to expand our study to include other categories of Wikipedia articles, such as evolving articles mentioned 
in Section 3.2. Due to time limitations, this thesis focused primarily on the stabilized and controversial categories. Consequently, the pool of articles in our study didn't contain a sizable amount of non-stabilized and non-controversial articles.

Comparative Study Of Quality Models. Third, we want to conduct a comparative study of a variety of quality models [33, 64, 47, 24] for each category. An experiment of this sort could provide further evidence for or against our idea of a 2-step quality assessment process. Such an experiment would involve implementing these models and reusing our existing data.

Analysis of Casual Reader Opinion. Last, but not least, we will investigate what affects the quality scores assigned to articles by casual readers. Our experimental study produced a variety of data (some of which had to be left out of this paper for space considerations) which can shed more light on how non-expert readers evaluate quality of information on-line. 


\section{Bibliography}

[1] Create Commons License Conditions. http://creativecommons.org/about/licenses.

[2] Javaxdelta Introduction. http://javaxdelta.sourceforge.net.

[3] Snowball. http://snowball.tartarus.org.

[4] Brockhaus Enzyklopädie. Brockhaus, Mannheim, 21 edition, 2007.

[5] Wikipedia Uncovered. PC Pro Magazine, August 2007.

[6] Wikipedia: Wissen fü alle. Stern, December 2007.

[7] Wikipedia vs. Encyclopedia A question of trust? Are online resources reliable or should we stick to traditional encyclopedias? Techradar.com, April 2008.

[8] D. Aha and D. Kibler. Instance-based learning algorithms. Machine Learning, 6:37-66, 1991.

[9] I. N. Athanasiadis, V. G. Kaburlasos, P. A. Mitkas, and V. Petridis. Applying machine learning techniques on air quality data for real-time decision support. In 1st Intl. NAISO Symposium on Information Technologies in Environmental Engineering (ITEE-2003), Gdansk, Poland, 2003. ICSC-NAISO Academic Press. Abstract in ICSC-NAISO Academic Press, Canada (ISBN:3906454339), pg.51.

[10] D.P. Ballou and Pazer H.L. Modeling data and process quality in multiinput, multi-output information systems. Management Science, 1985.

[11] Teresa Berry Barry X. Miller, Karl Helicher. I Want My Wikipedia! Library Journal, April 2006.

[12] George Bragues. Wiki-philosophizing in a marketplace of ideas Evaluating wikipeida's entries on seven great minds. Available at SSRN http //ssrn.com/abstract=978177, April 2007.

[13] Armand Brahaj. List of English Stop Words. http://armandbrahaj.blog.al/2009/04/14/list-of-english-stop-words/. 
[14] Gabriel De La Calzada and Alexander Dekhtyar. On Measuring the Quality of Wikipedia Articles. In WICOW'10: Proceedings of the 4th workshop on Information credibility on the web, New York, NY, USA, 2010. ACM.

[15] K.A. Clauson, H.H. Polen, M.N. Kamel Boulos, and J.H. Dzenowagis. Scope, completeness, and accuracy of drug information in Wikipedia. Ann Pharmacother, 2008.

[16] John G. Cleary and Leonard E. Trigg. K*: An instance-based learner using an entropic distance measure. In 12th International Conference on Machine Learning, pages 108-114, 1995.

[17] William W. Cohen. Fast effective rule induction. In Twelfth International Conference on Machine Learning, pages 115-123. Morgan Kaufmann, 1995.

[18] P. Denning, J. J. Horning, D. Parnas, and L. Weinstein. Wikipedia Risks. Communications of the ACM, 48(12), 2005.

[19] Apache Foundation. Apache lucene - overview. http://lucene.apache.org/java/docs.

[20] Y. Freund and L. Mason. The alternating decision tree learning algorithm. In Proceeding of the Sixteenth International Conference on $\mathrm{Ma}$ chine Learning, pages 124-133, Bled, Slovenia, 1999.

[21] Alexander Genkin, David D. Lewis, and David Madigan. Large-scale Bayesian logistic regression for text categorization. Technical report, DIMACS, 2004.

[22] Victor. Gijsbersm J. Ideals of Knowledge: Media from Plato to Wikipedia. Technical report, 2004.

[23] Jim Giles. Internet encyclopedias go head to head. Nature, December 2005.

[24] Daniel Hasan Dalip, Marcos André Gonçalves, Marco Cristo, and Pável Calado. Automatic quality assessment of content created collaboratively by web communities: a case study of wikipedia. In JCDL '09: Proceedings of the 9th ACM/IEEE-CS joint conference on Digital libraries, pages 295304, New York, NY, USA, 2009. ACM.

[25] Meiqun Hu, Ee-Peng Lim, Aixin Sun, Hady Wirawan Lauw, and Ba-Quy Vuong. Measuring article quality in wikipedia: models and evaluation. In CIKM '0\%: Proceedings of the sixteenth ACM conference on Conference on information and knowledge management, pages 243-252, New York, NY, USA, 2007. ACM. 
[26] Blumenstock J. Automatically Assessing the Quality of Wikipedia Articles. Technical report, UC Berkeley School of Information, 2008.

[27] Matthias Jarke and Yannis Vassiliou. Data Warehouse Quality: A Review of the DWQ Project. In In Proceedings of the Conference on Information Quality, 1997.

[28] S. S. Keerthi, S. K. Shevade, C. Bhattacharyya, and K. R. K. Murthy, 1999.

[29] Jon M. Kleinberg. Authoritative sources in a hyperlinked environment. Journal of the ACM, 46(5):604-632, September 1999.

[30] Ron Kohavi. The power of decision tables. In 8th European Conference on Machine Learning, pages 174-189. Springer, 1995.

[31] Niels Landwehr, Mark Hall, and Eibe Frank. Logistic model trees. 95(12):161-205, 2005.

[32] Yang W. Lee, Diane M. Strong, Beverly K. Kahn, and Richard Y. Wang. AIMQ: a methodology for information quality assessment. Inf. Manage., 40(2):133-146, 2002.

[33] Andrew Lih. Wikipedia as Participatory Journalism: Reliable Sources? Metrics for evaluating collaborative media as a news resource. In 5th International Symposium on Online Journalism, 2004.

[34] David J.C. Mackay. Introduction to gaussian processes, 1998.

[35] Vijay V. Mandke and Madhavan K. Nayar. Information integrity: A structure for its definition. In $I Q$, pages 314-338, 1997.

[36] Shannon McCaffrey. Political dirty-tricksters are using Wikipedia. Associated Press, http://www.msnbc.msn.com/id/12535412/, April 2006.

[37] Robert McHenry. The faith-based encyclopedia. Tech Central Station, http://www.tcsdaily.com/article.aspx?id=111504A, November 2004.

[38] MediaWiki. http //mediawiki.org.

[39] Rob Miller. Wikipedia Founder Jimmy Wales Responds. Slashdot, 2004.

[40] University of Waikato. Weka 3: Data Mining Software in Java. http://www.cs.waikato.ac.nz/ml/weka.

[41] Lawrence Page, Sergey Brin, Rajeev Motwani, and Terry Winograd. The PageRank Citation Ranking: Bringing Order to the Web. Technical Report 1999-66, Stanford InfoLab, November 1999. Previous number = SIDL-WP-1999-0120. 
[42] J. Platt. Machines using Sequential Minimal Optimization. In B. Schoelkopf, C. Burges, and A. Smola, editors, Advances in Kernel Methods - Support Vector Learning. MIT Press, 1998.

[43] Larry Press. Survey of Wikipedia accuracy and completeness. http //bpastudio.csudh.edu/fac/lpress/wikieval, 2006.

[44] R. Quinlan. Induction of decision trees. Machine Learning, 1(1):81-106, 1986.

[45] Ross Quinlan. C4.5: Programs for Machine Learning. Morgan Kaufmann Publishers, San Mateo, CA, 1993.

[46] Rainer Hammwöhner. Interlingual Aspects if Wikipedias Quality. In Mary Ann Robbert, M. Lynne Markus, and Barbara Klein, editors, 12th International Conference on Information Quality (ICIQ-2007). M.I.T., 2007. Universität Regensburg.

[47] Laura Rassbach, Trevor Pincock, and Brian Mingus. Exploring the feasibility of automatically rating online article quality. http://upload.wikimedia.org/wikipedia/wikimania2007/d/d3/ RassbachPincockMingus07.pdf, 2007.

[48] Jason D. Rennie, Lawrence Shih, Jaime Teevan, and David R. Karger. Tackling the Poor Assumptions of Naive Bayes Text Classifiers. In ICML, pages 616-623. AAAI Press, 2003.

[49] Peter J. Rousseeuw and Annick M. Leroy. Robust regression and outlier detection. John Wiley \& Sons, 1987.

[50] Hugo Steinhaus. Sur la division des corps matériels en parties. Bull. Acad. Polon. Sci. Cl. III. 4, pages 801-804, 1956.

[51] Marc Sumner, Eibe Frank, and Mark Hall. Speeding up logistic model tree induction. In 9th European Conference on Principles and Practice of Knowledge Discovery in Databases, pages 675-683. Springer, 2005.

[52] Vladimir N. Vapnik. The nature of statistical learning theory. SpringerVerlag New York, Inc., New York, NY, USA, 1995.

[53] Jakob Vob. Measuring Wikipedia. In Proceedings of the 10th International Conference of the International Society for Scientometrics and Infometrics, 2005.

[54] Simon Waldman. Who knows? The Guardian, http://www.guardian. co.uk/technology/2004/oct/26/g2.onlinesupplement, October 2004 . 
[55] Yair Wand and Richard Y. Wang. Anchoring data quality dimensions in ontological foundations. Commun. ACM, 39(11):86-95, 1996.

[56] Jun Wang, Zucker, and Jean-Daniel. Solving multiple-instance problem: A lazy learning approach. In Pat Langley, editor, 17th International Conference on Machine Learning, pages 1119-1125, 2000.

[57] Richard Y. Wang and Diane M. Strong. Beyond accuracy: what data quality means to data consumers. J. Manage. Inf. Syst., 12(4):5-33, 1996.

[58] G. Webb, J. Boughton, and Z. Wang. Not So Naive Bayes: Aggregating One-Dependence Estimators. Machine Learning, 58(1):5-24, 2005.

[59] Dorothee Wiegand. Entdeckungsreise. Digitale Enzyklopdien erklren die Welt. c't, March 2007.

[60] Wikipedia. English Edition, http //en.wikipedia.org.

[61] Wikipedia. http //en.wikipedia.org/wiki/Jimmy_Wales.

[62] Wikipedia. http //en.wikipedia.org/Wikipedia_reliability.

[63] Wikipedia. Wikipedia: Featured article criteria. http://en.wikipedia.org/wiki/Wikipedia Featured_article_criteria, November 2009.

[64] Honglei Zeng, Maher A. Alhossaini, Li Ding, Richard Fikes, and Deborah L. McGuinness. Computing trust from revision history. In PST, page 8, 2006.

[65] H. Zhang, L. Jiang, and J. Su. Hidden naive bayes. In Twentieth National Conference on Artificial Intelligence, pages 919-924. AAAI Press, 2005.

[66] Fei Zheng and Geoffrey I. Webb. Efficient lazy elimination for averagedone dependence estimators. In Proceedings of the Twenty-third International Conference on Machine Learning (ICML 2006), pages 1113-1120. ACM Press, 2006.

[67] Zijian Zheng and G. Webb. Lazy Learning of Bayesian Rules. Machine Learning, 4(1):53-84, 2000.

[68] R.W. Zmud. An empirical investigation of the dimensionality of the concept of information. Decision Sciences, 9(2), 1978. 


\section{Appendix A}

\section{Student Survey Results}

Results of the Wikipedia survey aggregated by article name are shown starting at Figure A. The result tables display the average and standard deviation values of student rating and confidence. The count column indicates the number of times an article has been successfully rated by a survey participant. Note that view count varies anywhere from 17 to 20 . Any discrepancies in article count are due to a participants not completing the survey and uneven coverage of articles to participants. 


\begin{tabular}{|c|c|c|c|c|c|}
\hline Name & avg(rating) & stddev(rating) & avg (conf) & stddev(conf) & count \\
\hline 2006 in music & 4.15 & 0.88 & 1.95 & 0.50 & 20 \\
\hline 4 chan & 4.00 & 0.50 & 1.53 & 0.66 & 18 \\
\hline Acianthera duartei & 1.45 & 0.55 & 1.23 & 0.37 & 20 \\
\hline Alan Turing & 4.50 & 0.36 & 1.74 & 0.57 & 19 \\
\hline Alexis Carrel & 3.68 & 0.69 & 1.20 & 0.43 & 20 \\
\hline Altoids & 3.36 & 0.76 & 1.97 & 0.26 & 18 \\
\hline B.A.T.M.A.N. & 3.00 & 0.74 & 1.18 & 0.33 & 19 \\
\hline Bizone (moth) & 1.38 & 0.44 & 1.35 & 0.53 & 20 \\
\hline Boards of Canada & 3.62 & 0.61 & 1.27 & 0.37 & 20 \\
\hline Btrfs & 3.58 & 0.69 & 1.35 & 0.48 & 20 \\
\hline Bubble sort & 3.68 & 0.71 & 1.82 & 0.65 & 19 \\
\hline $\begin{array}{ll}\text { California } & \text { Poly- } \\
\text { technic } & \text { State } \\
\text { University } & \end{array}$ & 4.11 & 0.79 & 2.53 & 0.30 & 19 \\
\hline Casio SK-1 & 3.18 & 0.94 & 1.65 & 0.54 & 17 \\
\hline Cat & 4.61 & 0.39 & 2.28 & 0.42 & 18 \\
\hline $\begin{array}{l}\text { CD-i games from } \\
\text { The Legend of } \\
\text { Zelda series }\end{array}$ & 4.11 & 0.80 & 1.61 & 0.58 & 19 \\
\hline Chair & 3.82 & 1.10 & 2.45 & 0.38 & 20 \\
\hline Chemical peel & 3.63 & 0.78 & 1.53 & 0.44 & 19 \\
\hline $\begin{array}{l}\text { Chen style tai chi } \\
\text { chuan }\end{array}$ & 4.08 & 0.62 & 1.12 & 0.27 & 20 \\
\hline Choi Jae-Soo & 2.42 & 0.77 & 1.44 & 0.57 & 18 \\
\hline $\begin{array}{l}\text { Declaration of the } \\
\text { Rights of Man and } \\
\text { of the Citizen }\end{array}$ & 3.95 & 0.65 & 1.53 & 0.47 & 19 \\
\hline Eric Hill & 1.98 & 0.91 & 1.12 & 0.27 & 20 \\
\hline Facebook & 4.55 & 0.58 & 2.45 & 0.39 & 19 \\
\hline $\begin{array}{l}\text { Fedora (operating } \\
\text { system) }\end{array}$ & 4.08 & 0.66 & 1.75 & 0.54 & 20 \\
\hline Filling station & 4.32 & 0.52 & 2.42 & 0.37 & 19 \\
\hline Fire Baptism & 2.18 & 0.57 & 1.13 & 0.22 & 19 \\
\hline $\begin{array}{l}\text { Fokker-Planck } \\
\text { equation }\end{array}$ & 3.95 & 0.77 & 1.27 & 0.37 & 20 \\
\hline Found art & 3.47 & 0.82 & 1.55 & 0.54 & 19 \\
\hline Gastropoda & 4.56 & 0.52 & 1.53 & 0.54 & 18 \\
\hline George Buchanan & 3.72 & 0.67 & 1.22 & 0.38 & 18 \\
\hline $\begin{array}{l}\text { George MacPher- } \\
\text { son }\end{array}$ & 3.16 & 0.83 & 1.13 & 0.27 & 19 \\
\hline Glass harmonica & 3.98 & 0.80 & 1.48 & 0.62 & 20 \\
\hline $\begin{array}{ll}\text { Global Workspace } \\
\text { Theory }\end{array}$ & 2.92 & 0.82 & 1.05 & 0.15 & 19 \\
\hline Google Wave & 3.87 & 0.51 & 1.76 & 0.61 & 19 \\
\hline Hermetia illucens & 2.68 & 0.68 & 1.18 & 0.29 & 17 \\
\hline Hip hop & 4.03 & 0.72 & 1.87 & 0.51 & 19 \\
\hline $\begin{array}{lll}\text { I Sette nani alla } \\
\text { riscossa }\end{array}$ & 1.35 & 0.92 & 1.18 & 0.43 & 20 \\
\hline Jared Graves & 2.38 & 1.02 & 1.30 & 0.37 & 20 \\
\hline
\end{tabular}

Table A.1: Survey Results 1 


\begin{tabular}{|c|c|c|c|c|c|}
\hline Name & avg(rating) & stddev(rating) & avg(conf) & stddev (conf) & count \\
\hline $\begin{array}{l}\text { John } \\
\text { son (Nova } \\
\text { politician) }\end{array}$ & 1.82 & 0.76 & 1.10 & 0.25 & 20 \\
\hline $\begin{array}{l}\text { Joseph Kokou Kof- } \\
\text { figoh }\end{array}$ & 3.25 & 1.03 & 1.19 & 0.38 & 18 \\
\hline K. Bhatnagar & 2.05 & 0.72 & 1.13 & 0.32 & 19 \\
\hline NASA & 4.50 & 0.47 & 2.02 & 0.49 & 20 \\
\hline Neural network & 3.80 & 0.81 & 1.52 & 0.43 & 20 \\
\hline $\begin{array}{l}\text { Never Gonna Give } \\
\text { You Up }\end{array}$ & 4.13 & 0.69 & 1.84 & 0.80 & 19 \\
\hline Nile & 4.29 & 0.35 & 1.88 & 0.44 & 17 \\
\hline Nintendocore & 2.67 & 0.69 & 1.50 & 0.55 & 18 \\
\hline Numerology & 3.68 & 0.67 & 1.74 & 0.50 & 19 \\
\hline Nvidia & 4.45 & 0.65 & 1.63 & 0.58 & 19 \\
\hline $\begin{array}{l}\text { Obusier de } 6 \text { pouces } \\
\text { Gribeauval }\end{array}$ & 2.36 & 0.62 & 1.58 & 0.48 & 18 \\
\hline $\begin{array}{l}\text { Paranormal Activ- } \\
\text { ity (film) }\end{array}$ & 4.17 & 0.76 & 1.67 & 0.62 & 18 \\
\hline $\begin{array}{l}\text { Peanut Corpora- } \\
\text { tion of America }\end{array}$ & 3.97 & 0.75 & 1.42 & 0.54 & 19 \\
\hline Pedro (film) & 3.67 & 0.50 & 1.39 & 0.54 & 18 \\
\hline Ravi Arimilli & 2.63 & 0.60 & 1.21 & 0.34 & 19 \\
\hline Religion & 4.38 & 0.47 & 2.20 & 0.37 & 20 \\
\hline Rogers Hornsby & 4.00 & 0.74 & 1.50 & 0.51 & 19 \\
\hline $\begin{array}{l}\text { San Luis Obispo, } \\
\text { California }\end{array}$ & 4.64 & 0.37 & 2.44 & 0.33 & 18 \\
\hline Seth Rogen & 4.39 & 0.35 & 2.03 & 0.44 & 19 \\
\hline Shortstop & 3.32 & 0.94 & 2.10 & 0.51 & 20 \\
\hline Signal averaging & 2.98 & 0.68 & 1.25 & 0.40 & 20 \\
\hline Slava River & 1.82 & 0.86 & 1.32 & 0.45 & 20 \\
\hline Spectre (comics) & 3.89 & 0.84 & 1.26 & 0.52 & 19 \\
\hline Stephen Colbert & 4.20 & 0.51 & 1.90 & 0.56 & 20 \\
\hline Streefkerk & 2.58 & 0.83 & 1.23 & 0.37 & 20 \\
\hline Suzuki method & 4.37 & 0.53 & 1.53 & 0.55 & 19 \\
\hline Tabata & 1.55 & 0.59 & 1.23 & 0.33 & 20 \\
\hline $\begin{array}{l}\text { The Audio Injected } \\
\text { Soul }\end{array}$ & 2.53 & 1.01 & 1.26 & 0.44 & 19 \\
\hline The Egyptian & 3.06 & 0.78 & 1.31 & 0.38 & 18 \\
\hline $\begin{array}{l}\text { The Horn of Mortal } \\
\text { Danger }\end{array}$ & 2.84 & 0.81 & 1.26 & 0.50 & 19 \\
\hline $\begin{array}{l}\text { The Seven Habits } \\
\text { of Highly Effective } \\
\text { People }\end{array}$ & 3.58 & 0.90 & 1.64 & 0.60 & 18 \\
\hline $\begin{array}{l}\text { This Earth of } \\
\text { Mankind }\end{array}$ & 3.47 & 0.83 & 1.18 & 0.37 & 19 \\
\hline Three Wolf Moon & 3.22 & 0.95 & 1.67 & 0.62 & 18 \\
\hline Thuy Trang & 3.44 & 0.86 & 1.56 & 0.51 & 17 \\
\hline Tiol-Oula & 1.34 & 0.59 & 1.34 & 0.54 & 19 \\
\hline Trans fat & 4.12 & 0.72 & 1.98 & 0.46 & 20 \\
\hline
\end{tabular}

Table A.2: Survey Results 2 


\begin{tabular}{|l|c|c|c|c|c|}
\hline Name & avg(rating) & stddev(rating) & avg(conf) & stddev(conf) & count \\
\hline Ununoctium & 4.00 & 0.79 & 1.55 & 0.50 & 20 \\
\hline User interface & 3.05 & 1.06 & 2.10 & 0.66 & 20 \\
\hline $\begin{array}{l}\text { USS Raleigh (CL- } \\
\text { 7) }\end{array}$ & 3.70 & 0.80 & 1.52 & 0.56 & 20 \\
\hline $\begin{array}{l}\text { Venkatraman } \\
\text { Ramakrishnan }\end{array}$ & 2.92 & 0.61 & 1.08 & 0.25 & 18 \\
\hline Windows 7 & 4.08 & 0.61 & 2.08 & 0.57 & 19 \\
\hline World of Warcraft & 4.40 & 0.49 & 2.05 & 0.65 & 20 \\
\hline YOYOW & 2.42 & 0.94 & 1.18 & 0.33 & 19 \\
\hline
\end{tabular}

Table A.3: Survey Results 3 